\title{
Trehalose-6-phosphate promotes fermentation and glucose repression in Saccharomyces cerevisiae
}

\author{
Rebeca L. Vicente ${ }^{1,2}$, Lucie Spina ${ }^{1}$, Jose P.L. Gómez ${ }^{1}$, Sebastien Dejean ${ }^{3}$, Jean-Luc Parrou ${ }^{1}$ and Jean Marie \\ François ${ }^{1,4 *}$ \\ ${ }^{1}$ LISBP; UMR INSA-CNRS 5504 \& INRA 792; Toulouse, France. \\ 2 Fundación Alfonso Martín Escudero; Madrid, Spain. \\ ${ }^{3}$ Institut de Mathématiques de Toulouse, 118 route de Narbonne, F-31062 Toulouse, France. \\ ${ }^{4}$ Toulouse White Biotechnology Center, UMS INSA-INRA-CNRS, F-31520 Ramonville. \\ * Corresponding Author: \\ Jean Marie François, LISBP INSA, 135 Avenue de Rangeuil, F-31077 Toulouse cedex 04; Phone: +33(0) 5622859 07; \\ E-mail:fran_jm@insa-toulouse.fr
}

ABSTRACT The yeast trehalose-6-phosphate synthase (Tps1) catalyzes the formation of trehalose-6-phosphate (T6P) in trehalose synthesis. Besides, Tps1 plays a key role in carbon and energy homeostasis in this microbial cell, as shown by the well documented loss of ATP and hyper accumulation of sugar phosphates in response to glucose addition in a mutant defective in this protein. The inability of a Saccharomyces cerevisiae tps1 mutant to cope with fermentable sugars is still a matter of debate. We reexamined this question through a quantitative analysis of the capability of TPS1 homologues from different origins to complement phenotypic defects of this mutant. Our results allowed to classify this complementation in three groups. A first group enclosed TPS1 of Klyveromyces lactis with that of S. cerevisiae as their expression in Sctps1 cells fully recovered wild type metabolic patterns and fermentation capacity in response to glucose. At the opposite was the group with TPS1 homologues from the bacteria Escherichia coli and Ralstonia solanacearum, the plant Arabidopsis thaliana and the insect Drosophila melanogaster whose metabolic profiles were comparable to those of a tps1 mutant, notably with almost no accumulation of T6P, strong impairment of ATP recovery and potent reduction of fermentation capacity, albeit these homologous genes were able to rescue growth of Sctps1 on glucose. In between was a group consisting of TPS1 homologues from other yeast species and filamentous fungi characterized by 5 to 10 times lower accumulation of T6P, a weaker recovery of ATP and a 3-times lower fermentation capacity than wild type. Finally, we found that glucose repression of gluconeogenic genes was strongly dependent on T6P. Altogether, our results suggest that the TPS protein is indispensable for growth on fermentable sugars, and points to a critical role of T6P as a sensing molecule that promotes sugar fermentation and glucose repression. doi: 10.15698/mic2018.10.651 Received originally: 14.05.2018; in revised form: 01.08.2018, Accepted 24.08.2018, Published 01.10.2018.

Keywords: TPS1, trehalose 6phosphate, glycolysis, flux sensing, Crabtree effect, glucose repression, Saccharomyces cerevisiae.
Abbreviations:
FBP - fructose-1,6-bisphosphate,
G6P - glucose-6-phosphate, T6P - trehalose-6-phosphate, TPS - T6P synthase.

\section{INTRODUCTION}

The yeast Saccharomyces cerevisiae is widely used as a eukaryotic model organism for fundamental research. In addition, it is the most utilized microorganism in traditional biotechnological applications such as bread-making and fermented drinks [1], and deserves a great desirability towards the exploitation of renewable carbon sources for fuels and commodity chemicals [2-5]. These applications often involve rational design of the carbon metabolic pathways to increase performance and robustness, quantitatively expressed as production titer, yield and productivity. This metabolic rewiring requires at first glance an extremely well detailed knowledge of the metabolic networks and their regulation.

Glycolysis is the backbone of the carbon metabolism in S. cerevisiae and even after more than 50 years of intensive 
research [6], the regulation of this pathway is not completely understood. A still unsolved issue concerns the role of the trehalose-6-phosphate synthase (TPS) complex in carbon and energy metabolism of the yeast $S$. cerevisiae. This complex contains four subunits, namely Tps1, Tps2, Tps3 and Tsl1. The subunit encoded by TPS1 catalyzes the formation of trehalose-6-phosphate (T6P) from glucose-6phosphate (G6P) and UDP-glucose. T6P is then dephosphorylated by the subunit encoded by TPS2. Subunits encoded by TPS 3 and TSL1 do not harbor any known catalytic function but may participate in the integrity of the TPS complex [7, 8]. The deletion of TPS1, not only prevents the synthesis of trehalose, an $\alpha$-D-glucopyranosyl-(1-> 1)-aD-glucopyranoside, that serves both as storage carbohydrate and stress protectant in yeast $[7,9]$, but most importantly, it leads to a growth defect on fermentable sugars $[8,10]$. This defect is witnessed by a massive accumulation of sugar phosphates and depletion of ATP upon addition of rapidly assimilated sugars to nutrient-starved yeast or yeast cultivated on non-fermentative carbon sources $[11,12]$. Two models have been proposed to account for this glycolytic defect phenotype, namely an over-activity of the major yeast hexokinase encoded by $H X K 2$ due to the loss of its inhibition by T6P [13], and a depletion of intracellular $\mathrm{Pi}$ due to hyper-accumulation of sugar phosphates leading to an inactive glyceraldehyde-3-P dehydrogenase [11]. More recently, van Heerden et al [14] revisited these two models through the combination of flux analysis using 13C labelling experiments and mathematical modelling. They concluded about the importance of Pi-dynamic in the startup of glycolysis, which can be brought about by an ATP hydrolysis, resulting from trehalose metabolic cycling. Nonetheless, this last model does not consider that a mutant defective in TPS2 does not exhibit any apparent glycolytic defects, except a very high accumulation of T6P [15]. In addition, besides this glycolytic defect, a yeast mutant defective in TPS1 exhibits other multiple and apparently unrelated physiological defects, such as hyper-accumulation of glycogen [16], sporulation deficiency [17], higher uncoupling of the respiratory chain [18], reduced phospholipid production [19], lack of gluconeogenesis repression [20], as well as a high sensitivity to temperature and oxidative stress [21]. Altogether, these results underline a wider role of Tps1 in the regulation of $S$. cerevisiae metabolism.

To further document on the importance of the Tps1 protein and its product T6P in yeast sugar metabolism, we decided to revisit previous works dealing with complementation studies of a $S$. cerevisiae tps $1 \Delta$ mutant (Sctps1 $\Delta$ ) with TPS1 homologues from different origins, including bacteria, fungi, plants and insects. This work was motivated by the fact that these complementation studies were merely qualitative, employing episomic plasmids in which TPS1 homologues were cloned either under their own promoter or under $A D H 1$ or PGK1 promoter. Also, these complementation assays were carried out with tps1 mutants from different strain backgrounds [22-27], which hampered any comparison between the data. In addition, a recent result of our group showed that $S$. cerevisiae tps1 $1 \Delta$ mutant recovered growth on glucose after transformation with the Yarrowia lipolytica TPS1 (YITPS1) cloned in an episomic plasmid under the PGK1 promoter, even though T6P was barely detectable [15]. Conversely, Bonini et al. [25] reported a partial glucose growth recovery of a Sctps $1 \Delta$ upon expression of the $E$. coli ots $A$ cloned in an episomic plasmid under PGK1 promoter while levels of T6P were almost comparable to those in wild type yeast. Therefore, in order to seek further on the role of Tps1 and T6P in yeast sugar fermentation, we compared the potency of various TPS1 homologues to quantitatively complement phenotypic traits of a tps $1 \Delta$ mutant made in the physiologically well characterized CEN.PK strain [28]. This comparative study was carried out by expressing these homologous genes on the same centromeric vector under a constitutive PGK1 promoter, instead of the TPS1 promoter, to avoid unpredicted transcriptional control of this gene. This work led us to propose that the Tps1 protein is indispensable for growth on a fermentable carbon source, whereas T6P may act as a sensing molecule promoting fermentation and glucose repression.

\section{RESULTS}

Rationale to select TPS1 homologues to complement the phenotype of the Sctps1 $\triangle$ mutant

Based on a phylogenetic analysis of the TPS proteins reported earlier by Avonce et al [29], we selected TPS1 genes that belong to the main branches of the tree topologies, and combined this selection with previous works reporting complementation of the Sctps $1 \Delta$ mutant by the selected genes. Accordingly, we performed a new phylogenetic analysis with the retained 14 TPS proteins. This analysis grouped these proteins into 3 subgroups (Fig. 1). In the first group are TPS proteins from the yeast species including S. cerevisiae, K. lactis, Candida albicans, Hansenula polymorpha and Pichia pastoris. A second subgroup comprises TPS from the yeast Schizosaccharomyces pombe and the two filamentous fungi Aspergillus nidulans and Magnaporthe grisea. The position of TPS from Yarrowia lipolyti$c a$ is somehow in forefront of these two subgroups. The TPS protein from the bacteria $E$. coli and $R$. solanacearum composed the third distinct subgroup, which also contains the protein from the insect $D$. melanogaster. The TPS protein from the plant $A$. thaliana encoded by TPS1 linked in between the second and the third subgroup. Comparison of amino acids sequence alignment of these TPS proteins confirmed that they all harbored the residues involved in the binding of the substrates G6P and UDPGIc (see Fig. S1). This alignment also revealed an $\mathrm{N}$-terminal extension of the $A$. thaliana TPS protein which is also present in the Rip protein of $R$. solanacearum (Fig. S2). It was shown that this $\mathrm{N}$-terminal extension has an inhibitory effect on the cataIytic activity of the TPS protein [30]. This alignment also underscores an important C-terminal extension of more than 300 amino acids length for the TPS protein of $A$. thaliana, P. pastoris and D. melanogaster. 


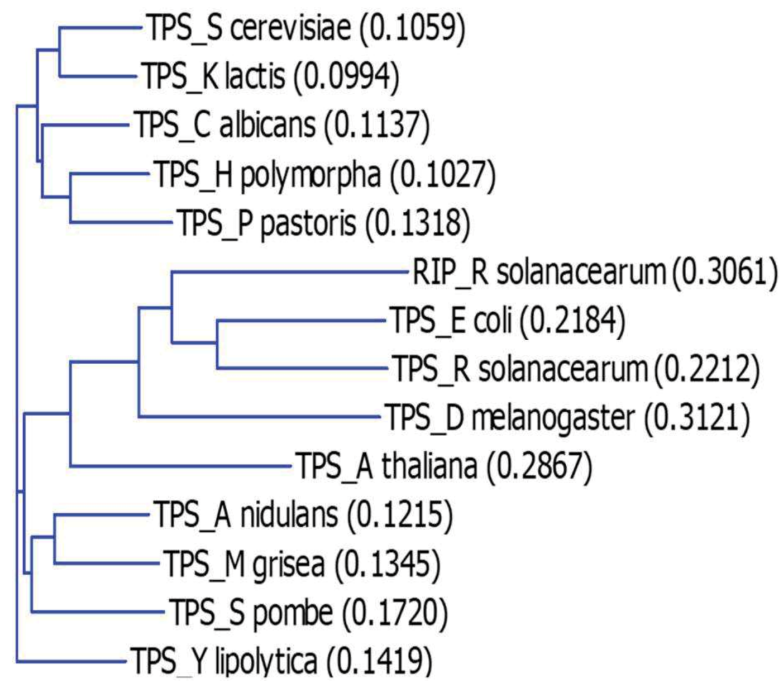

FIGURE 1: Phylogenetic tree of TPS protein homologues. Phylogenetic tree calculation is based on a sequence distance method and utilizes the Neighbour Joining algorithm of Saitou and Nei [31].

Striking differences in the potency of TPS1 homologues from different origins to recover growth of Sctps1 $\triangle$ on a fermentable sugar

To investigate the ability of TPS1 homologues from bacteria, fungi, plants and insects to complement phenotypes of S. cerevisiae tps1 $1 \Delta$ strain (Sctps1 1 ), we cloned their CDS (coding sequence) in the centromeric YCplac33 plasmid under the SCPGK1 promoter. As shown in Fig. 2, all the TPS1 homologous genes could complement the glucosenegative phenotype of a Sctps1 $1 \Delta$ mutant on solid media although with different efficiency. Indeed, the potency for growth recovery was lower with the plant, insect and $E$. coli TPS1 homologous genes than with yeast and filamen- tous fungi counterparts, and was only perceptible with the two $R$. solanacearum TPS1 homologous genes, namely ots $A$ and rip1. The weak capacity of these TPS1 homologues to complement the growth defect of Sctps1D could not be ascribed to a lack of gene expression, since transcripts of these genes were between 1 to 2,5-fold higher than that of the endogenous TPS1 in the wild type as determined by RT-qPCR (Fig. S3). It could be noticed that the transcription of TPS1 homologues from E. coli, A. thaliana and D. melanogaster was among the highest, in spite of a weaker capacity of these genes to complement the glucose growth defect of a Sctps1 $1 \Delta$.

We next measured the growth rate of Sctps $1 \Delta$ transformed with the different TPS1 homologues on different carbon sources. To this end, all yeast transformants were pre-cultured in the permissive YN medium containing $1 \%$ trehalose. As reported earlier, this culture condition mimicked a steady-state glucose limited continuous culture at low dilution rate which favors full respiration [32, 33] and can be quickly switched into a respiro-fermentative mode by addition of excess glucose [15]. When the cultures reached around 5 units $\mathrm{OD}_{600}$, they were diluted 50 -fold in $0.35 \mathrm{ml}$ of YN medium containing glucose (Fig. 3) or fructose (Fig. 4). This experiment clearly showed that TPS1 homologues do not equally restore growth of the Sctps1 $1 \Delta$ mutant on these fermentable sugars. With the exception of the $P$. pastoris TPS1, the TPS1 homologues of the yeast species were as efficient as the $S$. cerevisiae gene to reestablish growth rate of the Sctps $1 \Delta$ mutant on these sugars. In contrast, the resumption of growth was retarded in tps1 $1 \triangle$ expressing TPS1 homologues from the filamentous fungi $A$. nidulans and $M$. grisea. This delay in growth resumption was even more exacerbated upon expression of the E. coli, A. thaliana or D. melanogaster TPS1 homologue, with the extreme situation of Sctps $1 \Delta$ expressing $R$. solanacearum ots $A$ that only started to grow after $15 \mathrm{~h}$.

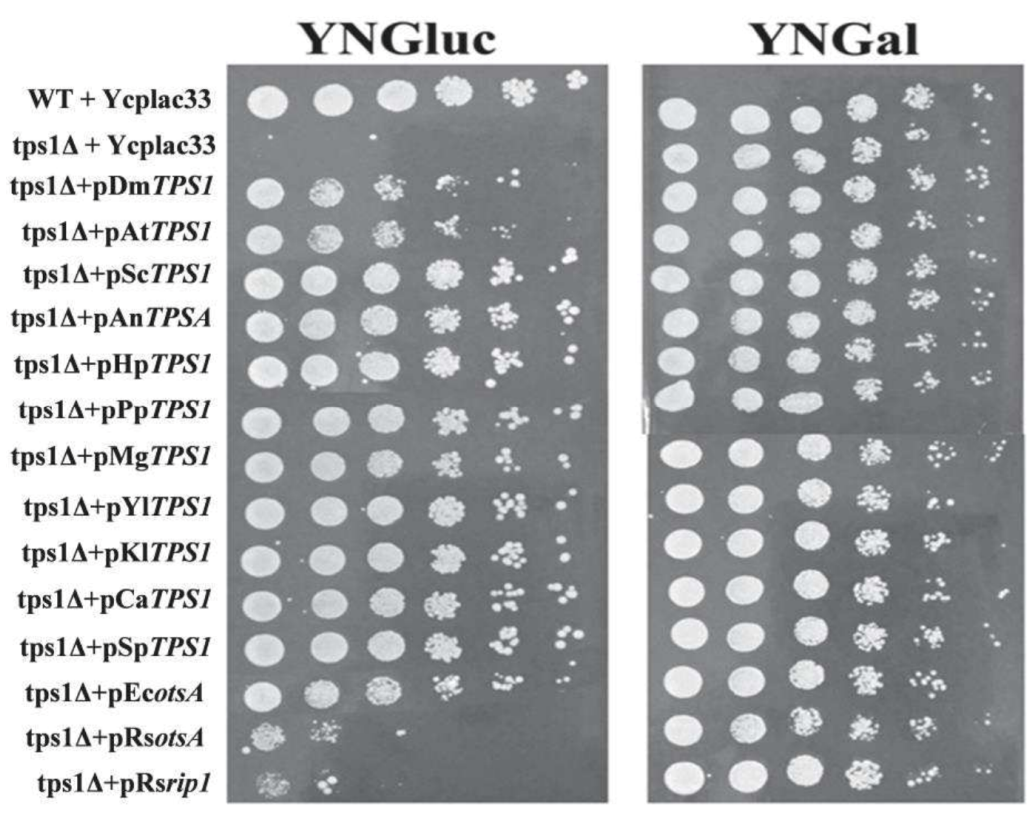

FIGURE 2: Growth assay of $S$. cerevisiae tps1 mutant transformed with TPS1 from different organisms on solid media. The Sctps1 $1 \Delta$ strain transformed with YcpLac33 plasmid bearing TPS1 homologue was cultivated on YN trehalose $1 \%$ till $5 \mathrm{OD}_{600}$ units. $10 \mu \mathrm{l}$ of serial dilution from this preculture was spotted on YN agar plate containing glucose or galactose at $2 \%(\mathrm{w} / \mathrm{v})$. Growth was scored after 2 days at $30^{\circ} \mathrm{C}$. 


\section{on YN Glucose}
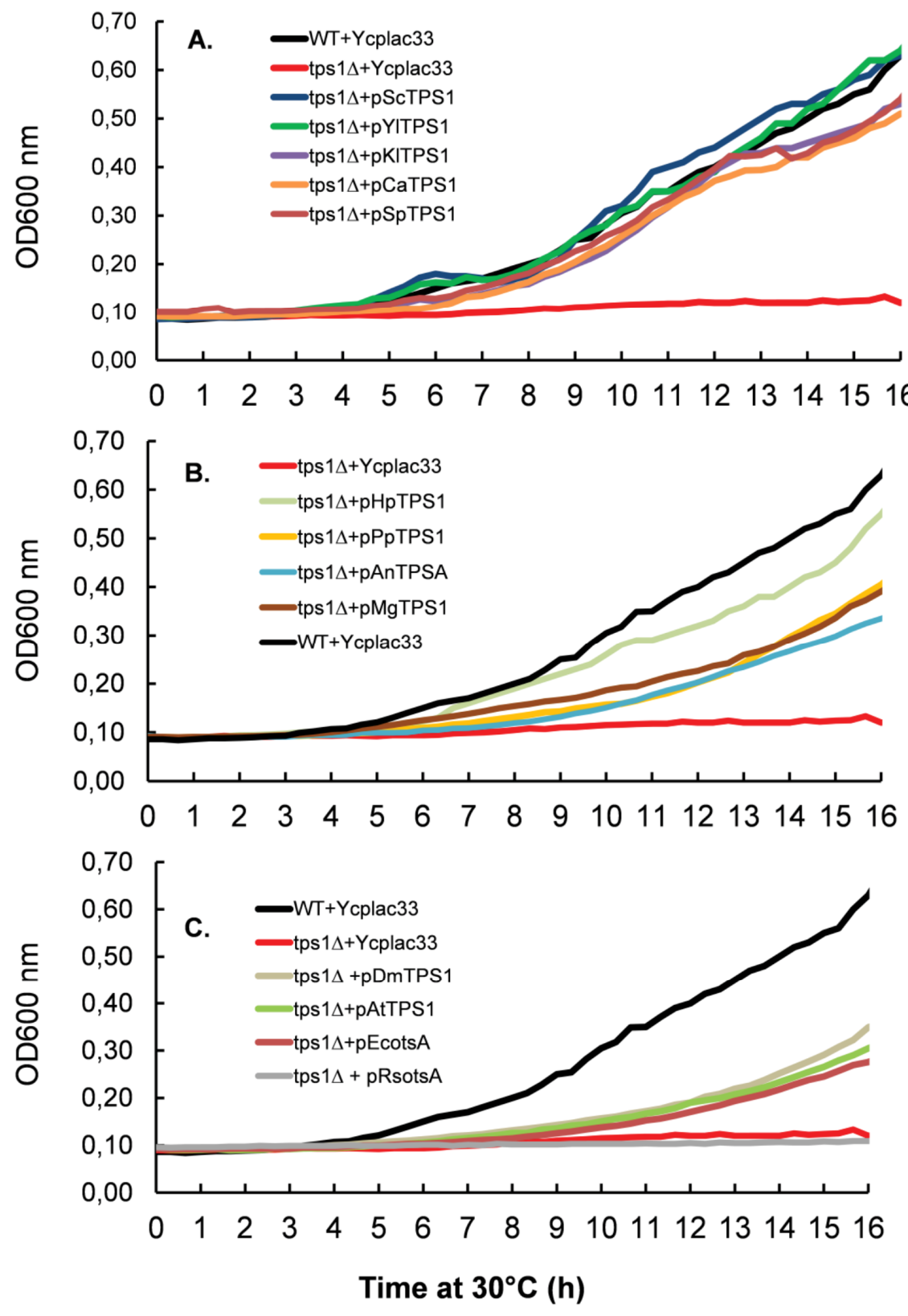

FIGURE 3: Resumption of growth of S. cerevisiae tps1 mutant transformed with TPS1 from different organism on YN glucose media in microtiter plate. (A) Complementation of sctps1 with TPS1 homologs from yeast species. (B) Complementation of Sctps1 with TPSD1 homologs from $P$. pastoris, H. polymorpha and the filamentous fungi $A$. niger and $M$. grisea. (C) Complementation of sctps1 with TPS1 homologs from bacteria, plants and drosophila. The experimental design was as in Figure 2 except that the yeast cells were inoculated in $250 \mu \mathrm{L}$ of $\mathrm{YN}$ containing glucose at $2 \%$ $(w / v)$. Results shown are the mean of two independent experiments. For the sake of clarity, standard deviation bars have been withdrawn, but overall the SD was in the range of $10-15 \%$.
Likewise, expression of Rsrip1, the other TPS1 homologue of this bacterium [34], as well as the tps1-156 allele, encoding for a catalytically dead Tps1 [35], failed to recover growth of Sctps1 $1 \Delta$ after two days of cultivation in glucose or fructose containing YN medium (data not shown). Notwithstanding, the difference in the capacity to resume growth on fermentable sugars, we verified whether Sctps1 1 transformed with the different TPS1 homologous genes exhibited also difference in their maximal growth rate $\left(\mu_{\max }\right)$. As reported in Table 1 , we found similar $\mu_{\max }$ on glucose and fructose as wild type of Sctps1 $1 \Delta$ complemented with yeast and filamentous fungi TPS1, with the exception of the $P$. pastoris and $M$. grisea gene for which the growth rate was $25-35 \%$ lower than that of wild type. Also, the maximal growth rate was more than two fold lower in
Sctps1 1 transformed with the TPS1 homologue from $E$. coli, $A$. thaliana and $D$. melanogaster. A generation time of $>10 \mathrm{~h}$ was estimated for the Sctps1D expressing RsotsA, whereas there was no significant growth of the Sctps1 $\Delta$ mutant bearing Rsrip1 on glucose or fructose. On the other hand, the growth resumption and maximal growth rate of Sctps1 $1 \Delta$ on the permissive carbon source galactose was not impacted by the expression of any of the different TPS1 homologues (Table 1 and supplementary data Fig. S4A). As a conclusion, these data indicated that the presence of the Tps1 protein is needed for growth on rapidly assimilated, fermentable sugars, and additionally suggest that the promptness at which the growth can restart on these sugars very likely requires a catalytic active Tps1 protein. 


\section{on YN Fructose}
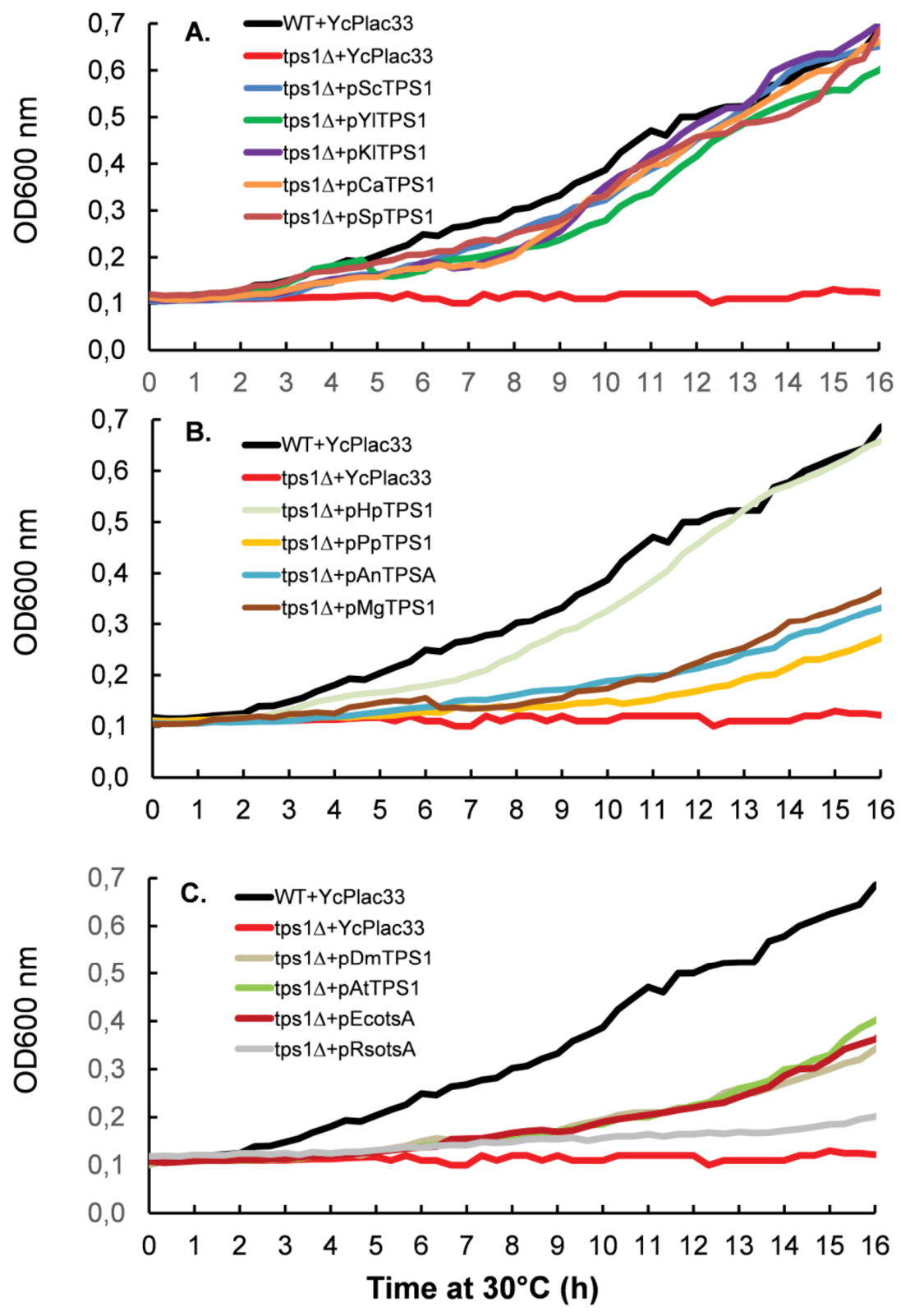

FIGURE 4: Resumption of growth of S. cerevisiae tps1 mutant transformed with TPS1 from different organism on YN fructose media in microtiter plate. (A) Complementation of sctps1 with TPS1 homologs from yeast species. (B) Complementation of Sctps1 with TPSD1 homologs from $P$. pastoris, $H$. polymorpha and the filamentous fungi $A$. niger and $M$. grisea. (C) Complementation of sctps1 with TPS1 homologs from bacteria, plants and drosophila. The experimental design was as in Fig. 2 except that the yeast cells were inoculated in $250 \mu \mathrm{L}$ of $\mathrm{YN}$ containing fructose at $2 \%(\mathrm{w} / \mathrm{v})$. For the sake of clarity, standard deviation bars have been withdrawn, but overall the SD was in the range of $10-15 \%$.
Tps1 activity in Sctps1 transformed with TPS1 homologues and correlation with trehalose levels

The disparity in the growth behavior of the Sctps1 $1 \Delta$ mutant complemented with TPS1 homologues of different origins while the expression of these genes was apparently correct (see Fig. S3), prompted us to verify whether this phenotypic complementation was accompanied by a measurable Tps1 activity, which would argue that the TPS proteins from these different origins are active in vivo in yeast ( $\mathrm{Ta}$ ble 2). A first indication that our strategy was valuable was given by the finding that Sctps $1 \Delta$ trans-

formed with the ScTPS1 gene expressed from a CEN plasmid under the PGK1 promoter regained the activity of Tps1 and levels of trehalose exactly as the wild type (Table 2). We also found that the Tps1 activity and trehalose levels in
Sctps1 $\Delta$ transformed with $K$. lactis, $H$. polymorpha, $A$. nidulans and $C$. albicans could be grouped together with that of wild type and Sctps1 $1 \triangle+$ pScTPS1. With the exception of the AnTPS1, this clustering matched the subgroup 1 of our phylogenetic analysis (Fig. 1). At the opposite, a second group which corresponded to cluster 2 in Fig. 1 was composed of Sctps1 $1 \Delta$ transformed with the TPS protein from the bacteria $E$. coli and $R$. solanacearum, the insect $D$. melanogaster and the plant $A$. thaliana. The activity of Tps1 measured in these transformants was overall ten times lower than that of the wild type. The third group, that also corroborated with the phylogenetic analysis, included TPS1 from S. pombe, $Y$. lipolytica, and $M$. grisea, and showed a Tps1 activity that was around five times lower than that of wild type but sufficiently high to accumulate high levels 
TABLE 1. Growth rate of S.cerevisiae tps1 mutant transformed with TPS1 from various origin in microplate on different carbon sources.

\begin{tabular}{|c|c|c|c|}
\hline Strain name & YN glucose & YN fructose & YN galactose \\
\hline WT+YcPlac33 & $0.29 \pm 0.02$ & $0.29 \pm 0.03$ & $0.24 \pm 0.03$ \\
\hline tps1 $1 \Delta+$ YCplac33 & NG & NG & $0.22 \pm 0.03$ \\
\hline $\operatorname{tps} 1 \Delta+p S c T P S 1$ & $0.29 \pm 0.02$ & $0.28 \pm 0.03$ & $0.26 \pm 0.03$ \\
\hline $\operatorname{tps} 1 \Delta+p S p T P S 1$ & $0.28 \pm 0.02$ & $0.29 \pm 0.03$ & $0.25 \pm 0.03$ \\
\hline $\operatorname{tps} 1 \Delta+p Y \mid T P S 1$ & $0.28 \pm 0.02$ & $0.29 \pm 0.03$ & $0.26 \pm 0.03$ \\
\hline $\operatorname{tps1\Delta +pKITPS1}$ & $0.29 \pm 0.02$ & $0.27 \pm 0.03$ & $0.25 \pm 0.03$ \\
\hline $\operatorname{tps} 1 \Delta+p C a T P S 1$ & $0.26 \pm 0.02$ & $0.25 \pm 0.03$ & $0.23 \pm 0.03$ \\
\hline $\operatorname{tps1\Delta +pPpTPS1}$ & $0.19 \pm 0.02$ & $0.15 \pm 0.03$ & $0.23 \pm 0.03$ \\
\hline $\operatorname{tps1\Delta +pHpTPS1}$ & $0.27 \pm 0.02$ & $0.29 \pm 0.03$ & $0.24 \pm 0.03$ \\
\hline $\operatorname{tps} 1 \Delta+p A n T P S 1$ & $0.26 \pm 0.02$ & $0.26 \pm 0.03$ & $0.24 \pm 0.03$ \\
\hline $\operatorname{tps} 1 \Delta+p M g T P S 1$ & $0.21 \pm 0.02$ & $0.22 \pm 0.03$ & $0.25 \pm 0.03$ \\
\hline $\operatorname{tps} 1 \Delta+p E \operatorname{cots} A$ & $0.14 \pm 0.02$ & $0.12 \pm 0.03$ & $0.24 \pm 0.03$ \\
\hline $\operatorname{tps} 1 \Delta+\mathrm{pRsots} A$ & $0.07 \pm 0.02$ & $0.08 \pm 0.03$ & $0.22 \pm 0.03$ \\
\hline tps1 $1+p R s r i p 1$ & $<0.05$ & $<0.05$ & $0.21 \pm 0.03$ \\
\hline $\operatorname{tps} 1 \Delta+\mathrm{pDm} T P S 1$ & $0.13 \pm 0.02$ & $0.12 \pm 0.03$ & $0.24 \pm 0.03$ \\
\hline $\operatorname{tps} 1 \Delta+p A t T P S A$ & $0.11 \pm 0.02$ & $0.13 \pm 0.03$ & $0.24 \pm 0.03$ \\
\hline
\end{tabular}

The yeast strains were cultivated in microtiter plates containing $350 \mu \mathrm{l}$ of culture media as described in Material and Methods. The microtiter plates were placed in a thermostated Elisa reader (Biotek) set at $30^{\circ} \mathrm{C}$ under smooth shaking and read every 15 min. NG: no growth. The values are the mean \pm standard deviation of three independent biological experiments.

of trehalose. Finally, the Tps1 activity in Sctps1 $1 \Delta$ expressing $P$. pastoris TPS1 was in the same range as that of the bacterial homologous gene (Table 2). When the trehalose content determined in the different strains was plotted as a function of Tps1 activities, a slightly sigmoidal curve was obtained (see supplementary Fig. S5A), allowing to evaluate that optimal synthesis of this disaccharide activity is obtained with an activity of Tps1 in the range of 30 nmol. min $^{-1}$. mg protein ${ }^{-1}$.

Sctps1 $1 \Delta$ complemented with different TPS1 exhibited different metabolite patterns in response to a glucose pulse

A well-established metabolic defect harbored by a Sctps1 $1 \Delta$ mutant is the huge accumulation of sugar phosphates, the rapid loss of ATP and the absence of T6P production in response to high glucose $[11,15,25,37,38]$. We therefore investigated the dynamic response of these metabolites to glucose in Sctps1D transformed with the different TPS1 homologues. We chose to present the metabolic data as heat map (Fig.5 and 6) since it provides a better visualization of the dynamic changes that occurred in the different strains and shall facilitate grouping of metabolic patterns according to the TPS1 homologue which complements the Sctps1 $1 \Delta$ mutant. Note that a classical time course representation of the data is reported in Supplementary Figures S6 to S10. We found, as expected, that the metabolic pattern of Sctps $1 \Delta$ complemented with its own gene was identical to that of the wild type strain and this pattern was also found with the Sctps1 $1 \Delta$ mutant transformed with the $K$. lactis TPS1, which is in line with the close phylogenetic proximity of these two proteins. At the opposite of this pattern, metabolic profiles of the Sctps $1 \Delta$ mutant expressing TPS1 from E. coli, R. solanacearum (otsA and rip1), 
TABLE 2. Activity of Trehalose 6-P synthase (Tps1) and levels of trehalose in the $S$. cerevisiae tps1 mutant transformed by TPS1 of various origins.

\begin{tabular}{|c|c|c|}
\hline Strain ID & $\begin{array}{c}\text { Tps1 activity } \\
\mathrm{nmol} /(\text { min.mg protein) }\end{array}$ & $\begin{array}{r}\text { Trehalose level } \\
(\mathrm{mg} / \mathrm{g} \mathrm{DCW})\end{array}$ \\
\hline WT+YCplac33 & $76.7 \pm 6.6$ & $106 \pm 13.5$ \\
\hline tps1 $1 \Delta+p S c T P S 1$ & $61.4 \pm 3.30$ & $93 \pm 15$ \\
\hline tps1 1 +pKITPS1 & $48.7 \pm 15.8$ & $97 \pm 9$ \\
\hline $\operatorname{tps} 1 \Delta+\mathrm{pCa} T P S 1$ & $25.3 \pm 3.3$ & $86 \pm 25$ \\
\hline tps1 1 +pHpTPS1 & $44.6 \pm 4.8$ & $50 \pm 14$ \\
\hline $\operatorname{tps} 1 \triangle+p A n T P S 1$ & $39.1 \pm 16.2$ & $87 \pm 28$ \\
\hline $\operatorname{tps} 1 \Delta+p S p T P S 1$ & $15.7 \pm 8.6$ & $75 \pm 12$ \\
\hline $\operatorname{tps} 1 \triangle+p M g T P S 1$ & $18.6 \pm 8.6$ & $45 \pm 13$ \\
\hline tps1 $1 \Delta+p Y \mid T P S 1$ & $8.3 \pm 3.1$ & $64 \pm 10$ \\
\hline $\operatorname{tps} 1 \Delta+p P p T P S 1$ & $5.9 \pm 1.8$ & $36 \pm 9$ \\
\hline $\operatorname{tps} 1 \Delta+p E \operatorname{cots} A$ & $5.40 \pm 3.8$ & $55 \pm 10$ \\
\hline $\operatorname{tps} 1 \Delta+p R s o t s A$ & $5.90 \pm 2.4$ & $1.1 \pm 0.15$ \\
\hline tps1 $1 \Delta+p R s r i p 1$ & $3.20 \pm 1.5$ & $7 \pm 1.5$ \\
\hline $\operatorname{tps} 1 \triangle+p D m T P S 1$ & $6.6 \pm 2.30$ & $15 \pm 11$ \\
\hline $\operatorname{tps} 1 \Delta+p A t T P S 1$ & $4.0 \pm 2.9$ & $1.5 \pm 0.5$ \\
\hline tps1 $\Delta+Y C p l a c 33$ & $<0.10$ & 0.00 \\
\hline
\end{tabular}

The activity of T6P synthase was measured at $42^{\circ} \mathrm{C}$ as described in Material and Methods. Results are the mean \pm SD of three independent assays. Trehalose was determined in stationary phase approximatively $12 \mathrm{~h}$ after glucose exhaustion from the medium, at a time when trehalose content is about to reach its maximum and stabilizes for several hours before consumption [36].

A. thaliana and D. melanogaster grouped together and were remarkably similar to that of the Sctps1D mutant. This second group was characterized by a failure to produce T6P (see also supplementary Figure S6) and by an accumulation of H6P (hexose-6-phosphate) and FBP (fructose-1,6-bisphosphate), which were three and then times higher, respectively, than in wild type in response to glucose. In addition, the recovery of ATP, which has been lost after glucose addition, was dramatically impaired in these transformants (Fig. 6 and Supplementary data in Fig. S8). This deficiency in ATP recovery was concomitant with the accumulation of high levels of inosine, which serves as a sink to avoid the cell to lose nucleotide pools [39].
At variance to the phylogenetic analysis reported in Fig. 1, the metabolic patterns exhibited by TPS1 homologues from other yeast species and filamentous fungi were rather dispersed into small subgroups, which can be related to either the wild type (group 1) or to the tps1 $\Delta$ profile (group 2). More specifically, the Sctps $1 \Delta$ mutant complemented with TPS1 homologues from $C$. albicans, S. pombe, and $H$. polymorpha and to a lesser extent with $A$. nidulans TPS1 presented a metabolic pattern in response to glucose which was similar to that of group 1 . The most notable differences were, nevertheless, an accumulation of T6P, which was four to ten times lower than in wild type, and a slower recovery of ATP following glucose addition. Probably as a consequence of this slower recovery of ATP, these strains were also characterized by a significant accumulation of inosine, which was slowly remobilized afterwards, leading to their clustering with the tps1 strain (group 2) for this specific metabolite (Fig. 5 and 6). On the other hand, the metabolic profile harbored by Sctps1 $1 \triangle$ expressing TPS1 from $Y$. lipolytica, $P$. pastoris and $M$. grisea is closer to group 2 than group 1, notably with respect to the very low levels of T6P, high accumulation $\mathrm{H} 6 \mathrm{P}$ and FBP as well as inosine, upon glucose addition, and as expected a very slow and delayed recovery of ATP. To conclude, these metabolic analyses provided a clear indication that the TPS protein from these different origins is not equally functional and even if the homologous gene can rescue growth on glucose, this does not necessarily imply the rebuilding of the wild type metabolite profile.

\section{T6P promotes rapid metabolic switch to fermentation}

When glucose is added to yeast cultivated on a respiratory carbon source, a rapid metabolic shift to fermentation is taking place. This shift is characterized by the production of ethanol and other fermentable byproducts such as glycerol and acetate. The rate at which these products are formed can be related to the fermentation capacity (see [40], for a definition). In agreement with its glucose growth defect phenotype, the loss of TPS1 function in the yeast $S$. cerevisiae almost eliminated the fermentation of glucose into ethanol (Fig.7A). The reintroduction of the wild type TPS1 in the tps1 $1 \Delta$ mutant as well as the $K$. lactis homologous gene fully recovered a wild type fermentation capacity. Also, expression of TPS1 from other yeast species $S$. pombe, C. albicans, $H$. polymorpha and $Y$. lipolytica as well as that of $A$. nidulans restored the glucose fermentation activity of a Sctps $1 \Delta$ mutant, but with a 15 to $30 \%$ reduced efficiency. By contrast, glucose consumption of the Sctps $1 \Delta$ mutant expressing the $E$. coli, R. solanacearum, A. thaliana and $D$. melanogaster TPS1 homologue was more than five times lower than in a wild type, and lower sugar consumption was accompanied by weaker production of ethanol. This data indicates that these yeast transformants hardly achieved their metabolic shift towards the fermentation. Likewise, but to a lesser extent, the fermentation activity was reduced by 3 to 4 -fold in Sctps $1 \Delta$ cells transformed with TPS1 homologues from $P$. pastoris and $M$. grisea. When plotting the fermentation capacity determined in all these yeast strains as a function of T6P measured five min 


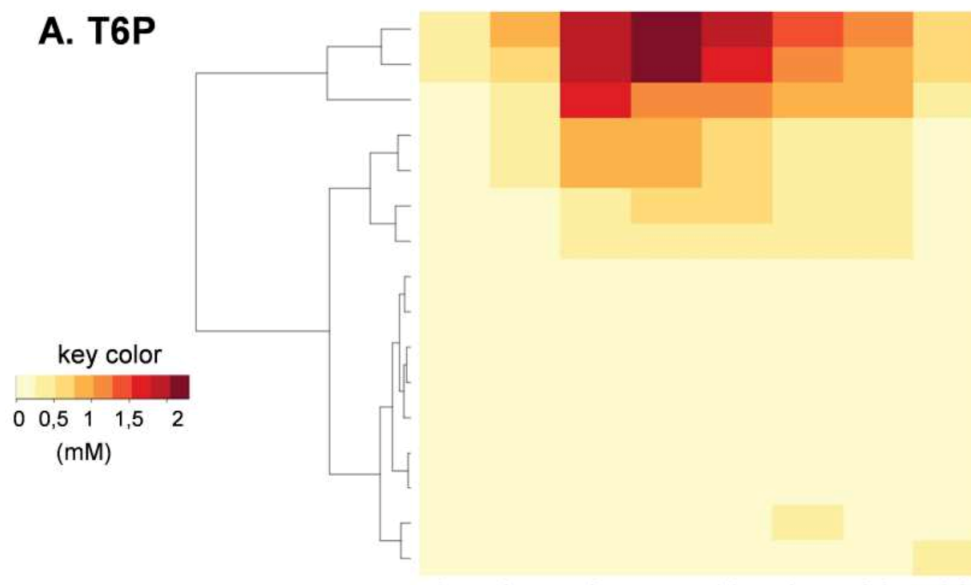

\section{B. $\mathrm{H} 6 \mathrm{P}$}

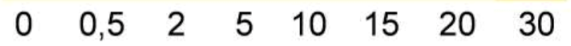

tps $1 \Delta+$ pScTPS 1 WT+YCpLac33 tps $1 \Delta+$ pKITPS1 tps $1 \Delta+$ pCaTPS 1 tps $1 \Delta+p S p T P S 1$ tps $1 \Delta+$ pHpTPS 1 tps $1 \Delta+$ pAnTPS 1 tps $1 \Delta+p P p T P S 1$ tps $1 \Delta+p E \operatorname{cots} A$ tps $1 \Delta+p$ Rsots $A$ tps $1 \Delta+p R s r i p 1$ tps $1 \Delta+$ pDmTPS 1 tps $1 \Delta+$ pAtTPS1 tps $1 \Delta+Y C p L a c 33$ tps $1 \Delta+p$ YITPS 1 tps $1 \Delta+$ pMgTPS1

tps $1 \Delta+p P p T P S 1$ tps $1 \Delta+$ pMgTPS 1 tps $1 \Delta+$ pRsrip 1 tps $1 \Delta+p$ YITPS 1 tps $1 \Delta+$ pAnTPS 1 tps1 $1 \Delta+Y C p L a c 33$ tps $1 \Delta+p E c o t s A$ tps $1 \Delta+p A t T P S 1$ tps $1 \Delta+p R$ sots $A$ tps $1 \Delta+$ pDmTPS 1 tps $1 \Delta+$ pHpTPS 1 tps $1 \Delta+p C a T P S 1$ tps $1 \Delta+$ pKITPS1 tps $1 \Delta+p S p T P S 1$ tps $1 \Delta+p S c T P S 1$ WT+YCpLac33
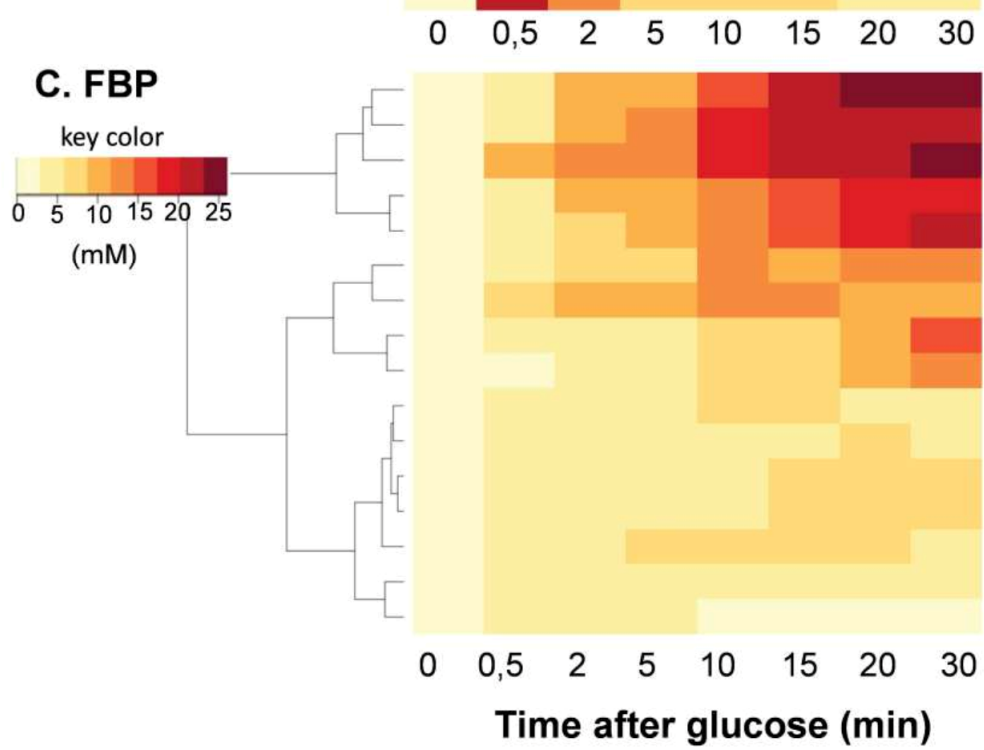

tps $1 \Delta+p A t T P S 1$ tps $1 \Delta+p E \operatorname{cots} A$ tps $1 \Delta+p R$ sots $A$ tps $1 \Delta+Y C p L a c 33$ tps $1 \Delta+p D$ DTPS1 tps $1 \Delta+$ pMgTPS1 tps $1 \Delta+p P p T P S 1$ tps $1 \Delta+p R$ srip 1 tps $1 \Delta+p$ YITPS1 tps $1 \Delta+$ pAnTPS 1 tps $1 \Delta+$ pHpTPS 1 tps $1 \Delta+$ pCaTPS 1 tps $1 \Delta+p S p T P S 1$ tps $1 \Delta+p S c T P S 1$ WT+YCpLac33 tps $1 \Delta+$ pKITPS1

\section{Time after glucose $(\mathrm{min})$}

FIGURE 5: Heat map representation of the change in T6P (A), H6P (B) and FBP (C) concentration in $S$. cerevisiae tps1 mutant expressing TPS1 from different organisms in response to a glucose pulse. The Sctps1 strain transformed with YCpL33 plasmid bearing TPS1 homologue was cultivated on YN Trehalose $1 \%$ till 5 $\mathrm{OD}_{600}$ units. Then, glucose (10 $\mathrm{g} / \mathrm{L}$ final concentration) was added and samples $(5 \mathrm{ml})$ at different times were rapidly collected by filtration for intracellular metabolite extraction and quantification. The data used for the heat map were the mean values of three independent experiments. Abbreviations: H6P mean hexose-6-Phosphate which is the sum of Glc6P and Fru6P; FBP means Fructose 1, 6bisphosphate. after glucose addition, we obtained a fairly acceptable Michaelis-Menten relationship, allowing to estimate that $50 \%$ of the fermentation capacity under these experimental conditions was obtained with about $0.2 \mathrm{mM}$ T6P (Fig. 7B). Likewise, a similar value was obtained by plotting the growth rate on glucose measured in these transformants as a function of T6P (supplementary data, Figure S5B).
Effective repression of gluconeogenic genes requires T6P It is well known that glucose triggers the transcriptional repression of the gluconeogenic genes FBP1 and PCK1 encoding fructose-1,6-biphosphate and phosphoenolpyruvate carboxykinase, respectively. While this repression requires phosphorylation of the sugar [41], the intracellular signal is still unknown [42]. Besides the inability of a $S$. cerevisiae 

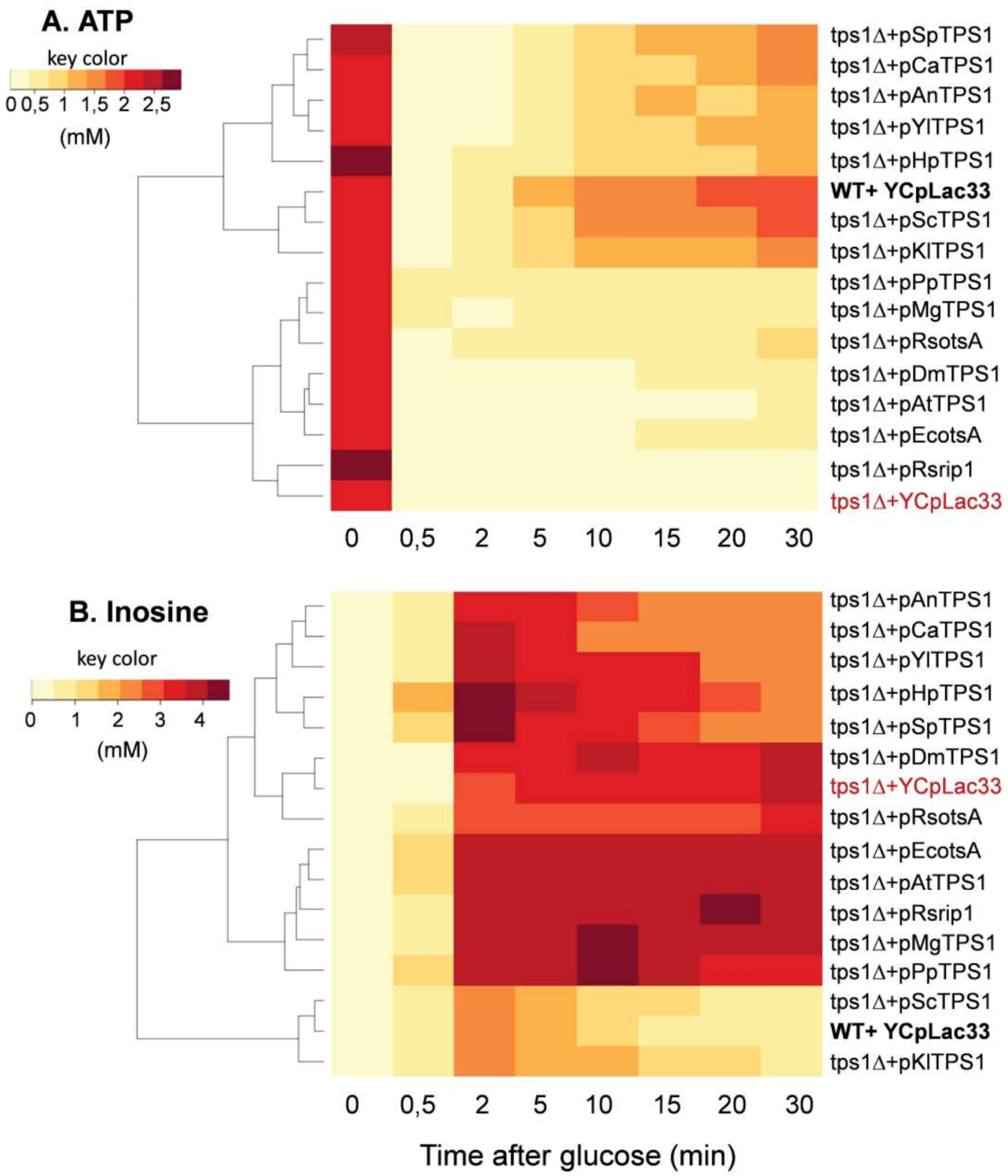

FIGURE 6: Heat map representation of the change of ATP (A) and inosine (B) concentration in $S$. cerevisiae tps1 mutant expressing TPS1 from different organisms in response to a glucose pulse. Same procedure as in Fig. 5. tps1 $\triangle$ mutant to grow on fermentable sugars, it was recently reported that $F B P 1$ and $P C K 1$ as well as genes of the glyoxylate pathway were not repressed upon glucose addition to this mutant cultivated on a respiratory carbon source [20]. Figure 8 shows a hyperbolic relationship that can be drawn by plotting the repression value for $F B P 1$ and $P C K 1$ genes determined $1 \mathrm{~h}$ after glucose addition and the concentration of T6P measured 5 min after the glucose pulse. Therefore, in addition to confirm the finding of these authors, we furthermore showed that the magnitude of the repression of these gluconeogenic genes is very likely dependent on the production of T6P. Interestingly, the T6P concentration that corresponded to half of the calculated maximal fold repression of $F B P 1$ and $P C K 1$ was in the range of $0.2-0.35 \mathrm{mM}$, which is in the same range of the value found for $50 \%$ of the maximal fermentation capacity (Fig. 7).

\section{DISCUSSION}

In this work, we revisited the capability of the S. cerevisiae TPS1 homologous genes from different origins to complement the metabolic defects of a Sctps $1 \Delta$ mutant strain. To realize this comparison in a quantitative manner, we selected TPS1 homologues harboring sufficient phylogenetic distances, and we cloned them into the centromeric plasmid YCpLac33 under the yeast PGK1 promoter. A first interesting result from this physiological analysis was to find that even in the absence of detectable T6P, the growth rescue of $t p s 1 \Delta$ on fermentable sugars was observed. This was demonstrated with Sctps1 $1 \triangle$ transformed with TPS1 homologues from bacteria, plant or insect that complemented the glucose-negative defect, albeit levels of T6P in these strains after glucose pulse was below our limit of detection. Should this observation mean that the TPS protein alone is sufficient to restore growth of Sctps1D on these sugars? We actually propose that a TPS protein that 
A.

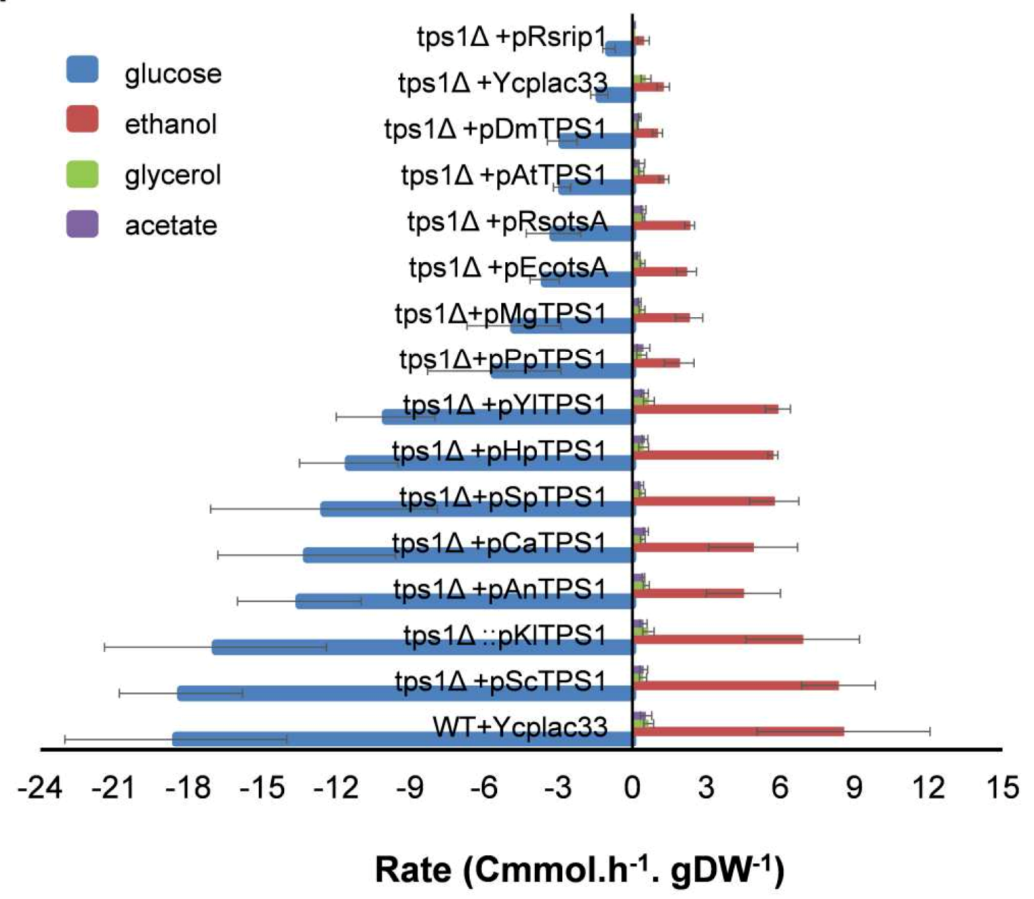

FIGURE 7: Determination of the fermentation capacity of $S$. cerevisiae tps 1 mutant complemented with TPS1 from different origins. The experimental design was as described in Fig.3 except that the supernatants of the cultures were taken at $5 \mathrm{~min}$ interval after glucose addition for $60 \mathrm{~min}$. Exo-metabolites determined by HPLC were expressed as $\mathrm{Cmmol}$ consumed (glucose) or produced (ethanol, acetate and glycerol) per $\mathrm{h}^{-1}$ and per $\mathrm{g}$ $\mathrm{DCW}^{-1}$. Results shown are the mean of three independent experiments with standard deviation bars (A). In (B), Relationship between fermentation capacity (FC) and T6P level. The Origin 2018 software (OriginLab, https://www.originlab.com/) was used to fit the data using the non-iterative fitting algorithm (Levenberg-Marquardt) selecting for the Michaelis-Menten or Hill models defined in the software.

B.

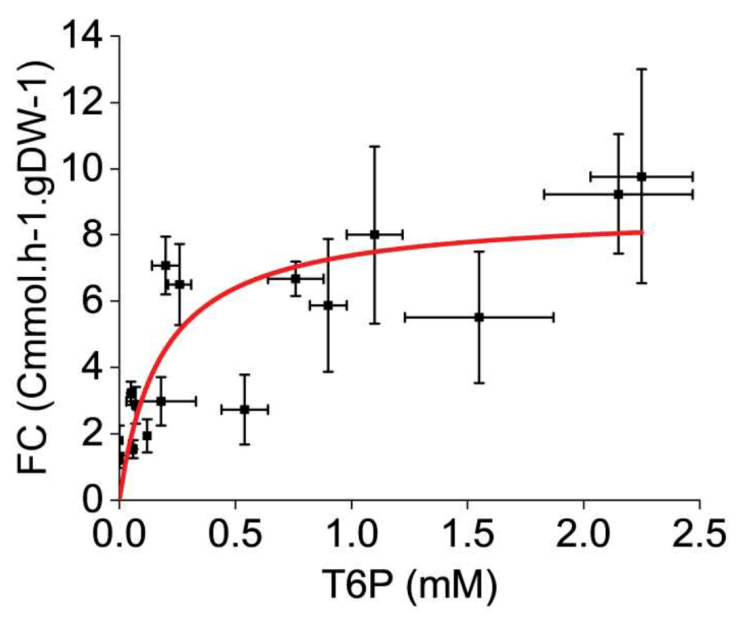

bears intact binding sites for G6P and UDPGlc is absolutely required for yeast to grow on a fermentable sugar, whereas T6P, the product of the reaction catalyzed by this protein, critically promotes sugar fermentation. This proposition is supported by the following data.

Firstly, Delorge et al. [43] reported that TPS genes from class II from the plant $A$. thaliana were unable to complement the glucose-negative phenotype of $\operatorname{Sctps} 1 \Delta$. They ascribed this failure to the lack of these enzymes to have catalytic activity due to the absence of the conserved binding sites for G6P and UDP-Glc in their amino acids sequence [44]. Also, Poueymiro et al. [34] showed that the catalytic inactive variant of $R$. solanacearum ripTPS ${ }^{\mathrm{Y} 154 \mathrm{~V}}$ (i.e the replacement of $Y$ at position 154 by $V$ abolishes binding of G6P) has lost its ability to complement a $S$. cerevisiae tps $1 \Delta t p s 2 \Delta$ mutant strain on glucose and fructose. Consistent with this result, we found that the growth defect of
Sctps $1 \Delta$ on glucose was not alleviated by the expression of a catalytically-dead Tps1 from the yeast $S$. cerevisiae (tps1156 variant that expresses a variant Tps1 in which the G6P binding is abolished by the replacement of $Y$ at position 156 by $\mathrm{V} ;$ [35]).

Secondly, it was reported that the growth recovery on glucose of the double tps $1 \Delta \operatorname{tps} 2 \Delta$ mutant strain was more effective than the single tps $1 \Delta$ mutant upon the expression of the A. thaliana TPS2 or TPS4 gene, which belong to the class I TPS-like proteins [43, 45]. A similar finding was shown for the $R$. solanacerum rip1 gene, which better complemented the glucose negative phenotype of a double tps $1 \Delta \operatorname{tps} 2 \Delta$ mutant than the single tps $1 \Delta$ mutant [34]. It was therefore suggested that the better growth recovery of a tps $1 \Delta t p s 2 \Delta$ mutant strain expressing these TPS homologs was due to the fact that they could accumulate even very low T6P levels in response to glucose, which was not 
the case for the single Sctps1 $\Delta$ strain $[34,43]$. From these reports, and with assumption that TPS proteins from these various species are expressed at the same level as that of the wild type yeast, an intriguing question is why they display so significant differences in their catalytic activity. In the case of Tps1 from A. thaliana encoded by AtTPS1, it was shown that the truncation of its extra $\mathrm{N}$-terminal extension, which is not present in the yeast protein, results in a ten to 40-fold increase of its in vitro activity [30]. The TPS protein of $R$. solanacearum encoded by rip1 also harbours an N-terminal extension, whose deletion might result in a similar effect. On the other hand, Tps1 from P. pastoris, $A$. thaliana and $D$. melanogaster show a long C-terminal extension which does not exist in any other TPS proteins. The impact of this C-extension on Tps1 activity has not been examined so far. Besides, taking into account that in vivo $S$. cerevisiae Tps1 is present in a complex with three others proteins, namely Tps2, Tps3 and Tsl1 [43, 46, 47], and that the optimal activity of Tps1 likely depends on the interaction with these partners [48], the very low activity of Tps1 from bacteria, plant and insects expressed in yeast might be explained by a failure to properly form this TPS complex. As a conclusion, a more precise structure-function analysis of the yeast Tps1 is awaiting to strengthen our hypothesis of a possible separated function of this protein in growth on fermentable sugar and in purely synthesis of T6P.

The finding that T6P promotes growth resumption on fermentable sugars coincided with the prompt recovery of ATP after its rapid fall following a glucose pulse. The reported inhibition of the major hexose phosphorylating enzyme encoded by HXK2 [13] could be invoked to account for this effect, because this inhibition would restrict the rate of the upper part of glycolysis which consumes ATP, allowing the lower part of the pathway to regenerate it. However, taken into consideration the inhibition constant of hexokinase II for T6P (i.e. 0.05 to $0.1 \mathrm{mM}[13,38]$ ), and the assumption that the intracellular glucose concentration is within 0.5 to $2 \mathrm{mM}$ in yeast cells challenged with glucose [49], the hexokinase activity would be maintained inhibited by more than $50 \%$ over more than $30 \mathrm{~min}$ after the glucose pulse, which would likely penalize the fast resumption of growth and the attendant fermentation activity. On the other hand, the probable absence of this inhibition in Sctps1 $1 \Delta$ cells expressing $S$. pombe, $Y$. lipolityca or A. nidulans TPS1 homologues due to their low level of T6P is inconsistent with the fact that the fermentation activity of these yeast strains was only 20 to $30 \%$ lower than that of a wild type strain.

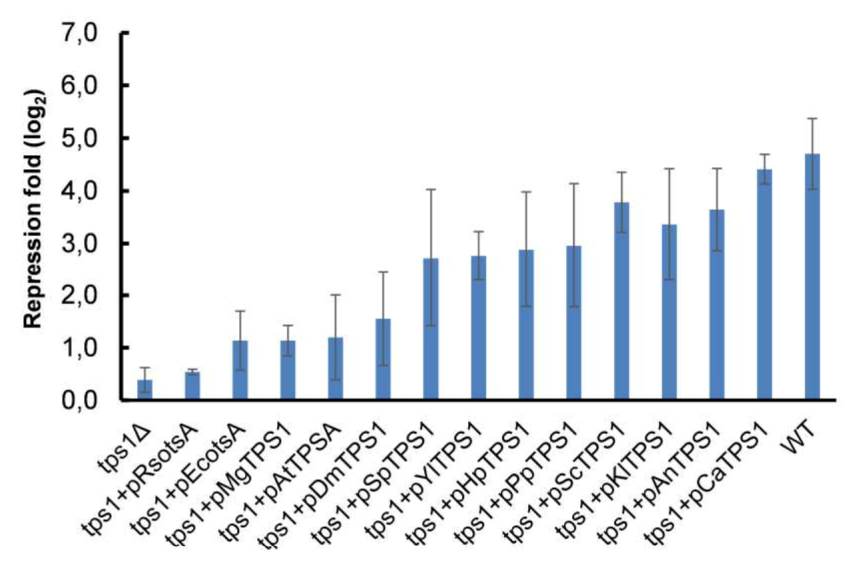

A. FBP1

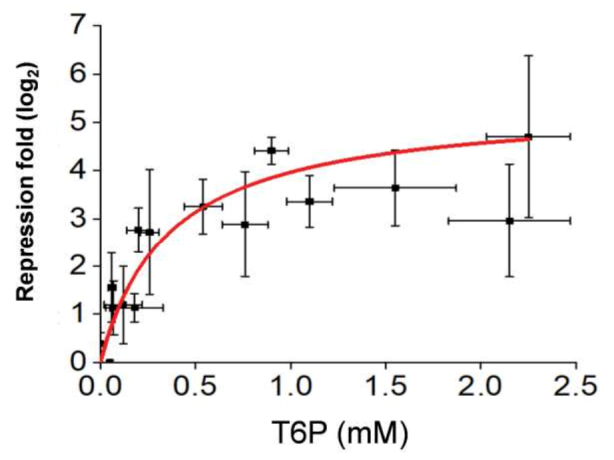

B. PCK1
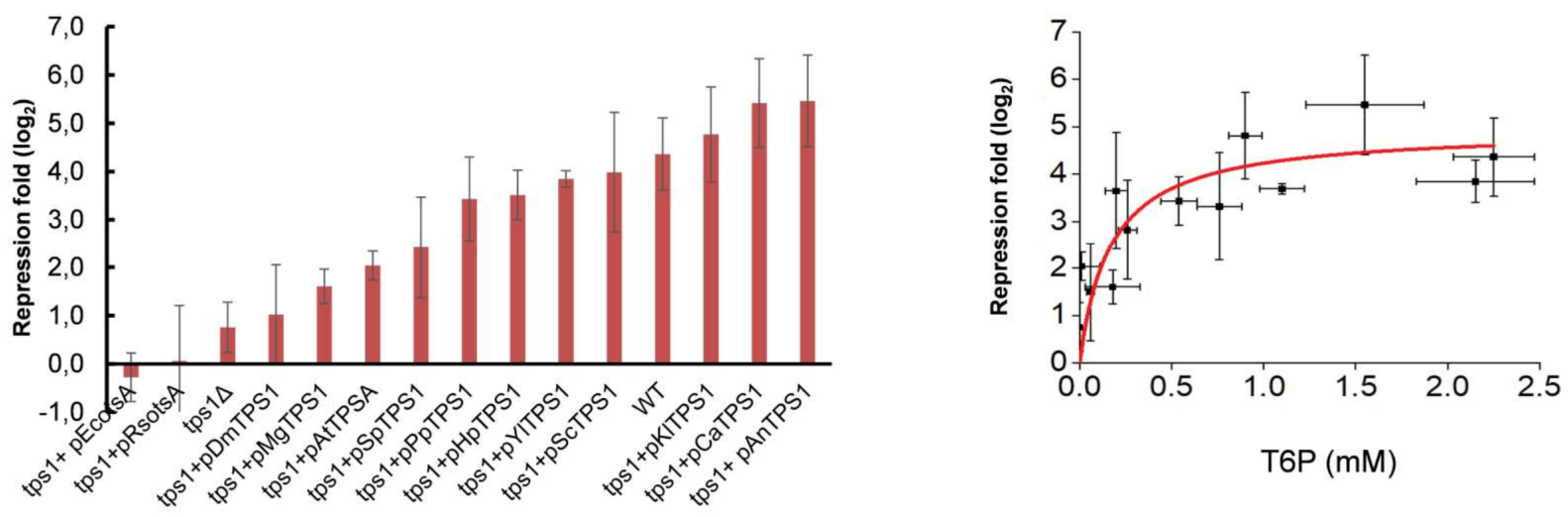

FIGURE 8: Expression level of FBP1 (A) and PCK1 (B) in S. cerevisiae tps1 mutant complemented with TPS1 from different organisms. The Sctps1 strains transformed with YCpL33 plasmid and with TPS1 homologues were cultivated on YN containing ethanol $2 \%$ until 5 units $\mathrm{OD}_{600}$. At this time, glucose (10 g/L final) was added and samples (15 OD units) were taken before and $1 \mathrm{~h}$ after glucose addition. The transcript levels of FBP1 and PCK1 was quantified by RT-qPCR using TAF10 and KRE11 as reference genes for normalization. Results shown are the mean of two. 
As a consequence, and in the light of previous work showing that manipulating the intracellular T6P content up or down has only little impact on the glucose influx into glycolysis $[15,25,38,48]$, one must invoke a complementary mechanism by which T6P promotes rapid growth on fermentable sugars. Interestingly, we found a hyperbolic correlation between fermentation capacity and levels of T6P, which is an indication that this metabolite is indeed a positive effector of fermentation. In addition, this data is consistent with the work of Wu et al. [50] which reported a parallel increase in the rate of glucose consumption, ethanol production and the rise of T6P following the short-term (50 to $300 \mathrm{sec}$ ) response of carbon-limited chemostat culture to a glucose pulse. Taken these results together, we postulate that T6P is implicated in the short-term Crabtree effect, which can be defined either by the immediate occurrence of aerobic fermentation in response to an excess of sugar to a sugar-limited yeast culture [51, 52], or more broadly as the capacity of yeast cells to readily switch from respiration to fermentation in response to glucose [53]. Although the molecular mechanism of this metabolic switch is still a matter of debate, it is clear that this switch is intimately dependent on the rate at which glucose enters the cell $[40,54,55]$. Heineman and colleagues [56] have introduced the notion of 'flux-sensing mechanism' to account for the fact that these metabolic phenotypes are triggered by a rapid change in sugar influx. Furthermore, they argued that these metabolic shifts require the cell to be able to measure "metabolic flux" through the level of some "flux-sensing" metabolites. FBP has been proposed as a possible flux-sensing molecule based on a good, yet strict, linear correlation between its intracellular levels and the sugar uptake rate [56]. In addition, this metabolite has been directly implicated in the Crabtree effect because of its ability to inhibit at physiological concentration (i.e. 2 to $10 \mathrm{mM}$ ) the oxidative phosphorylation of isolated mitochondria or permeabilized spheroplasts of yeast [57]. However, this latter data is not consistent with the fact that strains harbouring high levels of FBP such as Sctps1D expressing bacteria, plant or insect TPS protein hardly undergo the fermentation shift upon glucose addition. We therefore propose that T6P is a better flux-sensing molecule than FBP because it is metabolically located closer to the glucose uptake than FBP, it interacts at the gate of the glucose entrance through its action on hexokinase and it exhibits a Michaelis-Menten relationship with fermentation activity. The same flux-sensing function of T6P in glucose repression could be also invoked based on the fact that repression of the gluconeogenic genes FBP1 and $P C K 1$ is strictly dependent on T6P. Interestingly, the concentration of T6P that corresponded to $50 \%$ of the maximal glucose repression effect is in the same range (i.e. $0.2-0.3$ $\mathrm{mM}$ ) as the one estimated to reach $50 \%$ of the optimal fermentation activity, which suggests that the molecular targets of this metabolite are the same for these two processes.

The proposed role of T6P as a sugar signaling molecule in yeast is convergent with its assigned function in plants as a sensor of sucrose to integrate carbon assimilation with plant growth [58, 59]. This integration is obtained by the inhibition of the SNF1-related protein kinase by T6P. Whether this inhibition is direct or indirect is still unclear but, whatsoever the exact mechanism, this action of T6P overcomes the antagonistic effects of this kinase on the anabolic processes and growth in response to high level of sugars [60]. In yeast, the Snf1 kinase is essential in the derepression of gluconeogenic genes and in respiration [61]. However, contrary to expectation, this kinase is insensitive to T6P [20]. A common trait of yeast mutants harboring negligible levels of T6P upon glucose addition is their very lengthy recuperation of ATP, which could be explained not solely by a lack of hexokinase inhibition but also by the absence of a stimulatory action on downstream metabolic targets. In a previous work, we showed that the purine salvage pathway (PSP) was important in the control of ATP homeostasis under transition from respiration to fermentation and that the rebuilding of ATP shortly after a glucose pulse was largely attributed to the reassimilation of inosine [39]. Therefore, enzymes of the PSP can be potential targets of T6P. It is also conceivable that the failure of glucose repression of FBP1 and PCK1 is a consequence of the very low levels ATP in these strains which harbor low to negligible production of T6P.

\section{MATERIALS AND METHODS}

\section{Yeast strains, plasmids and transformation}

The Saccharomyces cerevisiae tps $1 \Delta$ and tps $2 \Delta$ mutants were derived from the CEN.PK133-5D ura3-52 strain [28] and have been described elsewhere $[15,62]$. The tps $1 \Delta$ strain was used as the host for transformation with the YCplac33 plasmid [63] bearing TPS1 from various origin (Saccharomyces cerevisiae, Drosophila melanogaster, Arabidopsis thaliana, Escherichia coli, Aspergillus nidulans, Hansenula polymorpha, Pichia pastoris, Magnaporthe grisea, Yarrowia lipolytica, Kluyveromyces lactis, Candida albicans, Schizosaccharomyces pombe and Ralstonia solanacearum) under the control of the $S$. cerevisiae PGK1 promoter. The TPS1 coding sequence from these organisms was amplified by PCR using $5^{\prime}$ and $3^{\prime}$ primers listed in Table S1. The PCR fragments were cloned into the YCplac33 plasmid using the In-Fusion system (Clontech), except the ones from $M$. grisea and $P$. pastoris, for which exons were amplified and assembled by using the NEBuilder ${ }^{\circledR}$ HiFi DNA Assembly system (New England Biolabs). All plasmid constructs bearing the TPS1 homologues are listed in Table 3 and were verified by DNA sequencing. The Lithium acetate procedure described in [64] was used to transform $S$. cerevisiae tps1 $1 \Delta$ mutant with the different plasmids.

\section{Culture conditions}

Unless otherwise stated, yeast cells were cultivated on a YN synthetic medium ( $17 \mathrm{~g}$ of yeast nitrogen base without amino acids and $5 \mathrm{~g}$ of ammonium sulfate per liter) buffered at $\mathrm{pH}$ 5.5 with $13.5 \mathrm{~g} / \mathrm{l}$ succinic acid and $6.5 \mathrm{~g} / \mathrm{I} \mathrm{NaOH}$, with $2 \%$ galactose as carbon source and complemented with $100 \mathrm{mg} / \mathrm{l}$ uracil. To verify that the tps $1 \Delta$ mutant has not undergone phenotypic or genetic 'reversion' that allows growth on glucose [14, 65], we streaked this strain on a YN fructose $2 \%$ complemented with uracil. Under this condition, no colonies were seen after at least 5 days of incubation at $30^{\circ} \mathrm{C}$, whereas several 
TABLE 3. List of plasmids used in this study.

\begin{tabular}{lll}
\hline Name & Description & Reference \\
\hline YCplac33 & CEN origin, URA3 marker & [61] \\
pScTPS1 & YCplac33 backbone with S. cerevisiae TPS1 gene & This study \\
pDmTPS1 & YCplac33 backbone with D. melanogaster Tps1 gene & This study \\
pAthTPS1 & YCplac33 backbone with A. thaliana TPS1 gene & This study \\
pAnTPS1 & YCplac33 backbone with A. nidulans tpsA gene & This study \\
pHpTPS1 & YCplac33 backbone with H. polymorpha TPS1 gene & This study \\
pPpTPS1 & YCplac33 backbone with P. pastoris TPS1 gene & This study \\
pMgTPS1 & YCplac33 backbone with M. grisea TPS1 gene & This study \\
pYITPS1 & YCplac33 backbone with Y. lipolytica TPS1 gene & This study \\
pKITPS1 & YCplac33 backbone with K. lactis TPS1 gene & This study \\
pCaTPS1 & YCplac33 backbone with C. albicans TPS1 gene & This study \\
pSpTPS1 & YCplac33 backbone with S. pombe TPS1 gene & This study \\
pEcotsA & YCplac33 backbone with E. coli otsA gene & This study \\
pRsotsA & YCplac33 backbone with R. solanacearum otsA gene & This study \\
pRsrip1 & YCplac33 backbone with R. solanacearum rip1 gene & This study
\end{tabular}

'colonies' arose after 2 days on YN glucose + uracile agar plates. Unless otherwise stated, growth of the mutant and transformants was carried out in baffled $1 \mathrm{~L}$ Erlenmeyer flasks containing $200 \mathrm{ml}$ of culture medium at $30^{\circ} \mathrm{C}$ on a rotary shaker at $200 \mathrm{rpm}$. The carbon source (glucose, galactose, raffinose) was added at $2 \%(\mathrm{w} / \mathrm{v})$ except trehalose which was added at $1 \%(\mathrm{w} / \mathrm{v})$. Growth on Petri dish plates was carried out in YN synthetic medium containing glucose or galactose at $2 \%$ $(\mathrm{w} / \mathrm{v})$ and supplemented with agar $2 \%(\mathrm{w} / \mathrm{v})$.

\section{Glucose pulse and intracellular metabolites determination} The glucose pulse experiment was performed as described in a previous report [39]. Briefly, the yeast cells were cultivated in YN containing $1 \%$ trehalose and buffered to $\mathrm{pH} 5.5$ using 50 $\mathrm{mM}$ potassium phthalate instead of Na-Succinate until they reached $\mathrm{OD}_{600} 5$ units (i.e. around $1 \mathrm{~g}$ dry mass per liter). Then, they were harvested, washed once with water at room temperature and resuspended in $\mathrm{YN}$ medium for $30 \mathrm{~min}$ at $30^{\circ} \mathrm{C}$. Then, glucose was added to a final concentration of $10 \mathrm{~g} / \mathrm{l}$ using a concentrated stock solution of $50 \%(w / v)$. Samples $(5$ $\mathrm{ml}$ ) were harvested at the indicated timepoints in the corresponding figures (Fig 4 to 6 ) by rapid filtration on $0.45 \mu \mathrm{m}$ porosity nitrocellulose filter and immediately quenched in $3-5$ $\mathrm{ml}$ of $75 \%$ hot ethanol, followed by $4 \mathrm{~min}$ of incubation in a water bath set at $80^{\circ} \mathrm{C}$ according to [66]. After extraction, removal of ethanol by evaporation and resuspension of the dry pellet in $0.5 \mathrm{ml}$ of sterile water, the metabolites were quantified in an ICS-3000 system equipped with an automatic eluent (KOH) generator system (RFIC, Dionex), an autosampler (AS50, Dionex), a photo diode array detector (Ultimate 3000, Dionex), a conductivity detector (part of ICS-3000, Dionex) and a mass-sensitive detector (MSQ Plus, Thermo Scientific) running in ESI mode (nitrogen pressure was 90 psi (1 psi=6.9 $\mathrm{kPa}$ ), capillary voltage was $3.5 \mathrm{kV}$ and probe temperature was $450{ }^{\circ} \mathrm{C}$ ), according to [39]. The concentration of intracellular metabolites is expressed in $\mathrm{mM}$ taking into account the assumption that $1 \mathrm{~g}$ of dry cell wall contains $2 \mathrm{ml}$ of cell sap [49].

\section{Determination of gene expression levels by RT-qPCR}

Quantification of TPS1, URA3 FBP1 and PCK1 transcripts was performed from two independent biological cultures. Yeast cells (about $15 \mathrm{OD}_{600}$ units) were collected by centrifugation (3000 rpm, $4^{\circ} \mathrm{C}, 2 \mathrm{~min}$ ), followed by a washing step with $1 \mathrm{ml}$ of sterilized water. The cell pellets were immediately frozen in liquid nitrogen and stored at $-80^{\circ} \mathrm{C}$. Cell disruption was performed with a Tissue Lyser system (Qiagen) at $30 \mathrm{~Hz}$ during 3 minutes. Total RNA was isolated according to RNeasy Total RNA Isolation Kit Manual (Qiagen) and concentrations of RNA samples were calculated by using NanoDrop 2000 UV-Vis spectrophotometer (Thermo Scientific, USA). Quality assessment of RNA samples was performed by Agilent RNA 6000 Nano kit and 2100 Bioanalyzer (Agilent Technologies, USA). Complementary DNA was synthetized with $1 \mu \mathrm{g}$ of total RNA using the iScript cDNA Synthesis Kit (Bio-Rad). The absence of genomic DNA in RNA samples was checked by qPCR before cDNA synthesis (minus RT control). A blank (No Template Control) was also incorporated in each assay. The Quantitative PCR ( $q P C R$ ) was performed using the Sso Advanced Universal SYBR Green system (Bio-Rad), $4 \mu$ of cDNA 10 times diluted and $2 \mu$ of primers ( $2 \mathrm{mM}$ ). The PCR program comprised an initial denaturation step of 1 minute at $95^{\circ} \mathrm{C}$ and amplification by 40 cycles of $10 \mathrm{sec}$ at $95^{\circ} \mathrm{C}, 45 \mathrm{sec}$ at $54^{\circ} \mathrm{C}$ in a Bio-Rad MyIQ cycler. The TAF1O and KRE11 genes were used as reference genes for normalization, following the recommendation of Teste et al. [67] for accurate transcripts measurement. Primer sequences used for RT- qPCR are provided in Table S2. 


\section{Assay of Trehalose-6P synthase (Tps1) activity}

The activity of TPS was determined in cell crude extracts according to the procedure described in [68]. Briefly, yeast cells (about $10 \mathrm{mg}$ dry mass or $50 \mathrm{OD}_{600}$ units) were collected by centrifugation ( $3 \mathrm{~min}$ at $2000 \mathrm{~g}, 4^{\circ} \mathrm{C}$ ) and washed with cold sterile water. Crude extract was performed by breaking yeast cells in $0.5 \mathrm{ml}$ extraction buffer $(20 \mathrm{mM}$ Hepes, pH 7.1 containing $1 \mathrm{mM}$ EDTA, $100 \mathrm{mM} \mathrm{KCl}, 1 \mathrm{mM} \mathrm{DTT}$, and $1 \mathrm{mM}$ PMSF) with $0.5 \mathrm{~g}$ glass beads ( $0.5 \mathrm{~mm}$ diameter; BioSpec 11079105) on a vortex set at full speed. The vortexing was done 6 times for 30 $\mathrm{sec}$ with $30 \mathrm{sec}$ intervals where tubes were kept on ice. The assay was realized at $42^{\circ} \mathrm{C}$ in the presence of $10 \mathrm{mM}$ Glucose$6-\mathrm{P} / 3 \mathrm{mM}$ Fructose-6-P and $5 \mathrm{mM}$ UDP-glucose in $20 \mathrm{mM}$ Hepes buffer, pH 7.1, 5 mM MgCl 2,1 mM EDTA, 100 mM KCl. The reaction was stopped by heat $\left(5 \mathrm{~min}\right.$ at $\left.80^{\circ} \mathrm{C}\right)$. The Tps 1 activity was determined either by the amount of UDP released or by the amount of trehalose-6-phosphate (T6P) produced and determined as intracellular metabolites by LC-MS as described above. Proteins were quantified with the Bradford reagent (Bio-Rad protein assay dye reagent concentrate, 5000006).

\section{Analytical methods}

Glucose, ethanol, acetate and glycerol were determined in the medium supernatant by withdrawing $1 \mathrm{ml}$ from the $\mathrm{YN}$ glucose cultures at the desired times. Samples were centrifuged at 13,000 rpm for $5 \mathrm{~min}$ in a bench-top centrifuge (Eppendorf 5415D), supernatants were filtered through a $0.45 \mu \mathrm{m}$ syringe filter, and stored at $-20^{\circ} \mathrm{C}$ until further analysis. Quantification was carried out using High performance liquid chromatography (HPLC) on an Ultimate 3000 system (Dionex, Sunnyvale, USA). The HPLC system was equipped with a cation-exchange column (Aminex HPX-87H - $300 \times 7.8 \mathrm{~mm}, 9 \mu \mathrm{m}$, Biorad), an autosampler (WPS-3000RS, Dionex), a RI detector (RID 10A, Shimadzu), and an UV/VIS detector (SPD-20A, Shimadzu). The mobile phase was $1.25 \mathrm{mM} \mathrm{H}_{2} \mathrm{SO}_{4}$ at a flow rate of $0.5 \mathrm{ml} / \mathrm{min}$. Column temperature was held at $35^{\circ} \mathrm{C}$. Glycogen and treha-

\section{REFERENCES}

1. Walker M (1998). Yeast physiology and Biotechnology. Wiley \& Sons, West Sussex, England.

2. Kondo A, Ishii J, Hara KY, Hasunuma T, Matsuda F (2013). Development of microbial cell factories for bio-refinery through synthetic bioengineering. J Biotechnol 163(2): 204-216. doi: 10.1016/j.jbiotec.2012.05.021

3. Eisenstein M (2016). Living factories of the future. Nature 531(7594): 401-403. doi: 10.1038/531401a

4. Siddiqui MS, Thodey K, Trenchard I, Smolke CD (2012). Advancing secondary metabolite biosynthesis in yeast with synthetic biology tools. FEMS Yeast Res 12(2): 144-170. doi: 10.1111/j.15671364.2011.00774.x

5. Mattanovich D, Sauer M, Gasser B (2014). Yeast biotechnology: teaching the old dog new tricks. Microb Cell Fact 13(1): 34. doi: 10.1186/1475-2859-13-34

6. Barnett JA, Entian KD (2005). A history of research on yeasts 9: regulation of sugar metabolism. Yeast 22(11): 835-894. doi: 10.1002/yea.1249

7. Francois J, Parrou JL (2001). Reserve carbohydrates metabolism in the yeast Saccharomyces cerevisiae. FEMS Microbiol Rev 25(1): 125145. doi: 10.1016/s0168-6445(00)00059-0 lose content were measured by glucose colorimetric assay after $\mathrm{Na}_{2} \mathrm{CO}_{3}$ treatment of the whole cell as described by [69].

\section{ACKNOWLEDGMENTS}

We thank colleagues of the JMF laboratory for critical discussions during this work and Dr Marion Schiavone for helping us with the Origin 2018 algorithm for fitting the kinetics data. This work was supported in part by ANR Blanc ${ }^{\circ}$ 07-2-200101 and by Region Midi Pyrénées (France), grant $\mathrm{N}^{\circ} 09003813$ to JMF. JLP is researcher at the Centre National de la Recherche Scientifique (CNRS), RLV was a fellow of the Fundacion Alfonso Martín Escudero and J.P.G.L is a postdoctoral fellow financially supported by Toulouse Region Midi Pyrenées.

\section{SUPPLEMENTAL MATERIAL}

All supplemental data for this article are available online at www.microbialcell.com.

\section{CONFLICT OF INTEREST}

The authors declare that they have no competing interests.

\section{COPYRIGHT}

(C) 2018 Vicente et al. This is an open-access article released under the terms of the Creative Commons Attribution (CC BY) license, which allows the unrestricted use, distribution, and reproduction in any medium, provided the original author and source are acknowledged.

Please cite this article as: Rebeca L. Vicente, Lucie Spina, Jose P.L. Gómez, Sebastien Dejean, Jean-Luc Parrou and Jean Marie François (2018). Trehalose-6-phosphate promotes fermentation and glucose repression in Saccharomyces cerevisiae. Microbial Cell 5(10): 444-459. doi: 10.15698/mic2018.10.651

8. Gancedo C, Flores CL (2004). The importance of a functional trehalose biosynthetic pathway for the life of yeasts and fungi. FEMS Yeast Res 4(4-5): 351-359. doi: 10.1016/s1567-1356(03)00222-8

9. Tapia $H$, Koshland DE (2014). Trehalose is a versatile and long-lived chaperone for desiccation tolerance. Curr Biol 24(23): 2758-2766. doi: 10.1016/j.cub.2014.10.005

10. Thevelein JM, Hohmann S (1995). Trehalose synthase: guard to the gate of glycolysis in yeast? Trends Biochem Sci 20(1): 3-10. doi: 10.1016/s0968-0004(00)88938-0

11. Van Aelst L, Hohmann S, Bulaya B, de KW, Sierkstra L, Neves MJ, Luyten K, Alijo R, Ramos J, Coccetti P, et al (1993). Molecular cloning of a gene involved in glucose sensing in the yeast Saccharomyces cerevisiae. Molecular Microbiology 8(5): 927-943. PMID: 8355617

12. Gonzalez MI, Stucka R, Blazquez MA, Feldmann H, Gancedo C (1992). Molecular cloning of CIF1, a yeast gene necessary for growth on glucose. Yeast 8(3): 183-192. doi:10.1002/yea.320080304

13. Blazquez MA, Lagunas R, Gancedo C, Gancedo JM (1993). Trehalose-6-phosphate, a new regulator of yeast glycolysis that inhibits hexokinases. FEBS Lett 329(1-2): 51-54. doi: 10.1016/00145793(93)80191-v 
14. van Heerden JH, Wortel MT, Bruggeman FJ, Heijnen JJ, Bollen YJ, Planque R, Hulshof J, O'Toole TG, Wahl SA, Teusink B (2014). Lost in transition: start-up of glycolysis yields subpopulations of nongrowing cells. Science 343(6174): 1245114. doi: 10.1126/science.1245114

15. Walther T, Mtimet N, Alkim C, Vax A, Loret MO, Ullah A, Gancedo C, Smits GJ, Francois JM (2013). Metabolic phenotypes of Saccharomyces cerevisiae mutants with altered trehalose 6-phosphate dynamics. Biochem J 454(2): 227-237. doi: 10.1042/BJ20130587

16. Cannon JF, Pringle JR, Fiechter A, Khalil M (1994). Characterization of glycogen-deficient g/c mutants of Saccharomyces cerevisiae. Genetics 136(2): 485-503. PMID: 8150278

17. Silva-Udawatta MN, Cannon JF (2001). Roles of trehalose phosphate synthase in yeast glycogen metabolism and sporulation. Mol Microbiol 40(6): 1345-1356. doi: 10.1046/j.1365-2958.2001.02477.x

18. Noubhani A, Bunoust O, Bonini BM, Thevelein JM, Devin A, Rigoulet $M$ (2009). The trehalose pathway regulates mitochondrial respiratory chain content through hexokinase 2 and CAMP in Saccharomyces cerevisiae. J Biol Chem 284(40): 27229-27234. doi: 10.1074/jbc.M109.029454

19. Hancock LC, Behta RP, Lopes JM (2006). Genomic analysis of the Opi- phenotype. Genetics 173(2): 621-634. doi: 10.1534/genetics.106.057489

20. Deroover S, Ghillebert R, Broeckx T, Winderickx J, Rolland F (2016). Trehalose-6-phosphate synthesis controls yeast gluconeogenesis downstream and independent of SNF1. FEMS Yeast Res 16(4). doi: 10.1093/femsyr/fow036

21. De Virgilio C, Hottiger T, Dominguez J, Boller T, Wiemken A (1994). The role of trehalose synthesis for the acquisition of thermotolerance in yeast. I. Genetic evidence that trehalose is a thermoprotectant. Eur J Biochem 219(1-2): 179-186. PMID:8306984

22. Blazquez MA, Stucka R, Feldmann H, Gancedo C (1994). Trehalose6-P synthase is dispensable for growth on glucose but not for spore germination in Schizosaccharomyces pombe. J Bacteriol 176(13): 3895-3902. doi: 10.1128/jb.176.13.3895-3902.1994

23. Blazquez MA, Santos E, Flores CL, Martinez-Zapater JM, Salinas J, Gancedo C (1998). Isolation and molecular characterization of the Arabidopsis TPS1 gene, encoding trehalose-6-phosphate synthase. Plant J 13(5): 685-689. doi: 10.1046/j.1365-313x.1998.00063.x

24. Reinders A, Romano I, Wiemken A, De Virgilio C (1999). The thermophilic yeast Hansenula polymorpha does not require trehalose synthesis for growth at high temperatures but does for normal acquisition of thermotolerance. J Bacteriol 181(15): 4665-4668. PMID:10419968

25. Bonini BM, Van Vaeck C, Larsson C, Gustafsson L, Ma P, Winderickx J, Van Dijck P, Thevelein JM (2000). Expression of escherichia coli otsA in a Saccharomyces cerevisiae tps1 mutant restores trehalose 6phosphate levels and partly restores growth and fermentation with glucose and control of glucose influx into glycolysis. Biochem J 350 (Pt1) 261-268. doi: 10.1042/0264-6021:3500261

26. Flores CL, Gancedo C, Petit T (2011). Disruption of Yarrowia lipolytica TPS1 gene encoding trehalose-6-P synthase does not affect growth in glucose but impairs growth at high temperature. PLoS ONE 6(9): e23695. doi: 10.1371/journal.pone.0023695

27. Ozer UE, Yucel M, Hamamci H (2016). Cloning and expression of trehalose-6-phosphate synthase 1 from Rhizopus oryzae. J Basic Microbiol 56(5): 459-468. doi: 10.1002/jobm.201500425

28. van Dijken JP, Bauer J, Brambilla L, Duboc P, Francois JM, Gancedo C, Giuseppin ML, Heijnen JJ, Hoare M, Lange HC, Madden EA, Niederberger $\mathrm{P}$, Nielsen J, Parrou JL, Petit T, Porro D, Reuss $\mathrm{M}$, van Riel N, Rizzi M, Steensma HY, Verrips CT, Vindelov J, Pronk JT (2000). An interlaboratory comparison of physiological and genetic properties of four Saccharomyces cerevisiae strains. Enzyme Microb Technol 26(910): 706-714. doi: 10.1016/s0141-0229(00)00162-9

29. Avonce N, Mendoza-Vargas A, Morett E, Iturriaga G (2006). Insights on the evolution of trehalose biosynthesis. BMC Evol Biol 6: 109. doi:10.1186/1471-2148-6-109

30. Van Dijck P, Mascorro-Gallardo JO, De Bus M, Royackers K, Iturriaga G, Thevelein JM (2002). Truncation of Arabidopsis thaliana and Selaginella lepidophylla trehalose-6-phosphate synthase unlocks high catalytic activity and supports high trehalose levels on expression in yeast. Biochem J 366(Pt 1): 63-71. doi: 10.1042/BJ20020517

31. Saitou N, Nei M (1987). The neighbor-joining method: a new method for reconstructing phylogenetic trees. Mol Biol Evol 4(4): 406425. doi: 10.1093/oxfordjournals.molbev.a040454

32. Jules M, Guillou V, Francois J, Parrou JL (2004). Two distinct pathways for trehalose assimilation in the yeast Saccharomyces cerevisiae. Appl Environ Microbiol 70(5): 2771-2778. doi:

10.1128/aem.70.5.2771-2778.2004

33. Jules M, Francois J, Parrou JL (2005). Autonomous oscillations in Saccharomyces cerevisiae during batch cultures on trehalose. FEBS J 272(6): 1490-1500. doi: 10.1111/j.1742-4658.2005.04588.x

34. Poueymiro M, Cazale AC, Francois JM, Parrou JL, Peeters N, Genin $S$ (2014). A Ralstonia solanacearum Type III Effector Directs the Production of the Plant Signal Metabolite Trehalose-6-Phosphate. MBio 5(6). doi: 10.1128/mBio.02065-14

35. Petitjean M, Teste MA, Francois JM, Parrou JL (2015). Yeast Tolerance to Various Stresses Relies on the Trehalose-6P Synthase (Tps1) Protein, Not on Trehalose. J Biol Chem 290(26): 16177-16190. doi: 10.1074/jbc.M115.653899

36. Francois J, Neves MJ, Hers HG (1991). The control of trehalose biosynthesis in Saccharomyces cerevisiae: evidence for a catabolite inactivation and repression of trehalose-6-phosphate synthase and trehalose-6-phosphate phosphatase. Yeast 7(6): 575-587. doi: 10.1002/yea.320070605

37. Neves MJ, Hohmann S, Bell W, Dumortier F, Luyten K, Ramos J, Cobbaert P, de KW, Kaneva Z, Thevelein JM (1995). Control of glucose influx into glycolysis and pleiotropic effects studied in different isogenic sets of Saccharomyces cerevisiae mutants in trehalose biosynthesis. Curr Genet 27(2): 110-122. doi: 10.1007/bf00313424

38. Hohmann S, Bell W, Neves MJ, Valckx D, Thevelein JM (1996). Evidence for trehalose-6-phosphate-dependent and -independent mechanisms in the control of sugar influx into yeast glycolysis. Mol Microbiol 20(5): 981-991. doi: 10.1111/j.1365-2958.1996.tb02539.x

39. Walther T, Novo M, Rossger K, Letisse F, Loret MO, Portais JC, Francois JM (2010). Control of ATP homeostasis during the respirofermentative transition in yeast. Mol Syst Biol 6: 344. doi: 10.1038/msb.2009.100

40. Hagman A, Sall T, Piskur J (2014). Analysis of the yeast short-term Crabtree effect and its origin. FEBS J 281(21): 4805-4814. doi: 10.1111/febs.13019

41. Yin Z, Smith RJ, Brown AJ (1996). Multiple signalling pathways trigger the exquisite sensitivity of yeast gluconeogenic mRNAs to glucose. Mol Microbiol 20(4): 751-764. doi: 10.1111/j.13652958.1996.tb02514.x

42. Gancedo JM (2008). The early steps of glucose signalling in yeast. FEMS Microbiol Rev 32(4): 673-704. doi: 10.1111/j.15746976.2008.00117.x

43. Delorge I, Figueroa CM, Feil R, Lunn JE, Van DP (2015). Trehalose6-phosphate synthase 1 is not the only active TPS in Arabidopsis thaliana. Biochem J 466(2): 283-290. doi: 10.1042/BJ20141322 
44. Gibson RP, Tarling CA, Roberts S, Withers SG, Davies GJ (2004). The donor subsite of trehalose-6-phosphate synthase: binary complexes with UDP-glucose and UDP-2-deoxy-2-fluoro-glucose at $2 \mathrm{~A}$ resolution. J Biol Chem 279(3): 1950-1955. doi:

\subsection{4/jbc.M307643200}

45. Vandesteene L, Lopez-Galvis L, Vanneste K, Feil R, Maere S, Lammens W, Rolland F, Lunn JE, Avonce N, Beeckman T, Van Dijck P (2012). Expansive evolution of the trehalose-6-phosphate phosphatase gene family in Arabidopsis. Plant Physiol 160(2): 884-896. doi: 10.1104/pp.112.201400

46. Londesborough J, Vuorio OE (1993). Purification of trehalose synthase from baker's yeast. Its temperature-dependent activation by fructose 6-phosphate and inhibition by phosphate. Eur J Biochem 216(3): 841-848. doi: 10.1111/j.1432-1033.1993.tb18206.x

47. Bell W, Klaassen P, Ohnacker M, Boller T, Herweijer M, Schoppink $P$, Van der ZP, Wiemken A (1992). Characterization of the $56-k D a$ subunit of yeast trehalose-6-phosphate synthase and cloning of its gene reveal its identity with the product of CIF1, a regulator of carbon catabolite inactivation. Eur J Biochem 209(3): 951-959. doi: 10.1111/j.1432-1033.1992.tb17368.x

48. Van Vaeck C, Wera S, Van Dijck P, Thevelein JM (2001). Analysis and modification of trehalose 6-phosphate levels in the yeast Saccharomyces cerevisiae with the use of Bacillus subtilis phosphotrehalase. Biochem J 353(Pt 1): 157-162. doi: 10.1042/0264-6021:3530157

49. Teusink B, Diderich JA, Westerhoff HV, van Dam K, Walsh MC (1998). Intracellular glucose concentration in derepressed yeast cells consuming glucose is high enough to reduce the glucose transport rate by 50\%. J Bacteriol 180(3): 556-562. PMID:9457857

50. Wu L, van Dam J, Schipper D, Kresnowati MT, Proell AM, Ras C, van Winden WA, van Gulik WM, Heijnen JJ (2006). Short-term metabolome dynamics and carbon, electron, and ATP balances in chemostatgrown Saccharomyces cerevisiae CEN.PK 113-7D following a glucose pulse. Appl Environ Microbiol 72(5): 3566-3577. doi:

10.1128/AEM.72.5.3566-3577.2006

51. Pronk JT, Yde SH, van Dijken JP (1996). Pyruvate metabolism in Saccharomyces cerevisiae. Yeast 12(16): 1607-1633. doi: 10.1002/(SICI)1097-0061(199612)12:16<1607::AID-YEA70>3.0.CO;2-4

52. Dashko S, Zhou N, Compagno C, Piskur J (2014). Why, when, and how did yeast evolve alcoholic fermentation? FEMS Yeast Res 14(6): 826-832. doi: 10.1111/1567-1364.12161

53. De Deken RH (1966). The Crabtree effect: a regulatory system in yeast. J Gen Microbiol 44(2): 149-156. doi: 10.1099/00221287-44-2149

54. Vemuri GN, Eiteman MA, McEwen JE, Olsson L, Nielsen J (2007). Increasing NADH oxidation reduces overflow metabolism in Saccharomyces cerevisiae. Proc Natl Acad Sci U S A 104(7): 2402-2407. doi: 10.1073/pnas.0607469104

55. Hagman A, Piskur J (2015). A Study on the Fundamental Mechanism and the Evolutionary Driving Forces behind Aerobic Fermentation in Yeast. PLoS ONE 10(1): e0116942. doi:

10.1371/journal.pone.0116942

56. Huberts DH, Niebel B, Heinemann M (2012). A flux-sensing mechanism could regulate the switch between respiration and fermentation. FEMS Yeast Res 12(2): 118-128. doi: 10.1111/j.1567-

1364.2011.00767.x

57. Diaz-Ruiz R, Averet N, Araiza D, Pinson B, Uribe-Carvajal S, Devin A, Rigoulet $M$ (2008). Mitochondrial oxidative phosphorylation is regulated by fructose 1,6-bisphosphate. A possible role in Crabtree effect induction? J Biol Chem 283(40): 26948-26955. doi: 10.1074/jbc.M800408200

58. Lawlor DW, Paul MJ (2014). Source/sink interactions underpin crop yield: the case for trehalose 6-phosphate/SnRK1 in improvement of wheat. Front Plant Sci 5: 418. doi: 10.3389/fpls.2014.00418

59. Schluepmann H, Berke L, Sanchez-Perez GF (2012). Metabolism control over growth: a case for trehalose-6-phosphate in plants. J Exp Bot 63(9): 3379-3390. doi: 10.1093/jxb/err311

60. Zhang Y, Primavesi LF, Jhurreea D, Andralojc PJ, Mitchell RA, Powers SJ, Schluepmann H, Delatte T, Wingler A, Paul MJ (2009). Inhibition of SNF1-related protein kinase1 activity and regulation of metabolic pathways by trehalose-6-phosphate. Plant Physiol 149(4): 1860-1871. doi: 10.1104/pp.108.133934

61. Gancedo JM (1998). Yeast carbon catabolite repression. Microbiol Mol Biol Rev 62(2): 334-361. PMID:9618445

62. Guillou V, Plourde-Owobi L, Parrou JL, Goma G, Francois J (2004). Role of reserve carbohydrates in the growth dynamics of Saccharomyces cerevisiae. FEMS Yeast Res 4(8): 773-787. doi:

10.1016/j.femsyr.2004.05.005

63. Gietz RD, Sugino A (1988). New yeast-Escherichia coli shuttle vectors constructed with in vitro mutagenized yeast genes lacking sixbase pair restriction sites. Gene 74(2): 527-534. doi: 10.1016/03781119(88)90185-0

64. Gietz RD, Schiestl RH (2007). High-efficiency yeast transformation using the LiAc/SS carrier DNA/PEG method. Nat Protoc 2(1): 31-34. doi: $10.1038 /$ nprot. 2007.13

65. Blazquez MA, Gancedo C (1994). Identification of extragenic suppressors of the cif1 mutation in Saccharomyces cerevisiae. Curr Genet 25(2): 89-94. doi: 10.1007/bf00309531

66. Gonzalez B, Francois J, Renaud M (1997). A rapid and reliable method for metabolite extraction in yeast using boiling buffered ethanol. Yeast 13(14): 1347-1355. doi: 10.1002/(SICI)10970061(199711)13:14<1347::AID-YEA176>3.0.CO;2-O

67. Teste MA, Duquenne M, Francois JM, Parrou JL (2009). Validation of reference genes for quantitative expression analysis by real-time RT-PCR in Saccharomyces cerevisiae. BMC Mol Biol 10: 99. doi: 10.1186/1471-2199-10-99

68. Vandercammen A, Francois J, Hers HG (1989). Characterization of trehalose-6-phosphate synthase and trehalose-6-phosphate phosphatase of Saccharomyces cerevisiae. Eur J Biochem 182(3): 613-620. doi: 10.1111/j.1432-1033.1989.tb14870.x

69. Parrou JL, Francois J (1997). A simplified procedure for a rapid and reliable assay of both glycogen and trehalose in whole yeast cells58. Anal Biochem 248(1): 186-188. doi: 10.1006/abio.1997.2138

70. Gibson RP, Turkenburg JP, Charnock SJ, Lloyd R, Davies GJ (2002). Insights into trehalose synthesis provided by the structure of the retaining glucosyltransferase OtsA. Chem Biol 9(12): 1337-1346. doi: 10.1016/s1074-5521(02)00292-2

71. Thompson JD, Higgins DG, Gibson TJ (1994). CLUSTAL W: improving the sensitivity of progressive multiple sequence alignment through sequence weighting, position-specific gap penalties and weight matrix choice. Nucleic Acids Res 22(22): 4673-4680. doi:

10.1093/nar/22.22.4673

72. Teste MA, Duquenne M, Francois JM, Parrou JL (2009). Validation of reference genes for quantitative expression analysis by real-time RT-PCR in Saccharomyces cerevisiae. BMC Mol Biol 10:99. doi: 10.1186/1471-2199-10-99 